\title{
Categorical Biases in Human Occipitoparietal Cortex
}

\author{
๑Edward F. Ester, ${ }^{1}$ ㅈThomas C. Sprague, ${ }^{2}$ and ${ }^{\circ}$ John T. Serences ${ }^{3}$ \\ ${ }^{1}$ Department of Psychology, Center for Complex Systems and Brain Sciences, and FAU Brain Institute, Florida Atlantic University, Boca Raton, \\ Florida 33431, ${ }^{2}$ Department of Psychological and Brain Sciences, University of California, Santa Barbara, California 93106, and ${ }^{3}$ Department of Psychology, \\ Neurosciences Graduate Program, and Kavli Institute for Brain and Mind, University of California, San Diego, California 92093
}

Categorization allows organisms to generalize existing knowledge to novel stimuli and to discriminate between physically similar yet conceptually different stimuli. Humans, nonhuman primates, and rodents can readily learn arbitrary categories defined by low-level visual features, and learning distorts perceptual sensitivity for category-defining features such that differences between physically similar yet categorically distinct exemplars are enhanced, whereas differences between equally similar but categorically identical stimuli are reduced. We report a possible basis for these distortions in human occipitoparietal cortex. In three experiments, we used an inverted encoding model to recover population-level representations of stimuli from multivoxel and multielectrode patterns of human brain activity while human participants (both sexes) classified continuous stimulus sets into discrete groups. In each experiment, reconstructed representations of to-be-categorized stimuli were systematically biased toward the center of the appropriate category. These biases were largest for exemplars near a category boundary, predicted participants' overt category judgments, emerged shortly after stimulus onset, and could not be explained by mechanisms of response selection or motor preparation. Collectively, our findings suggest that category learning can influence processing at the earliest stages of cortical visual processing.

Key words: categorization; EEG; fMRI; human; occipital cortex

\section{Significance Statement}

Category learning enhances perceptual sensitivity for physically similar yet categorically different stimuli. We report a possible mechanism for these changes in human occipitoparietal cortex. In three experiments, we used an inverted encoding model to recover population-level representations of stimuli from multivariate patterns in occipitoparietal cortex while participants categorized sets of continuous stimuli into discrete groups. The recovered representations were systematically biased by category membership, with larger biases for exemplars adjacent to a category boundary. These results suggest that mechanisms of categorization shape information processing at the earliest stages of the visual system.

\section{Introduction}

Categorization refers to the process of mapping continuous sensory inputs onto discrete and behaviorally relevant concepts. It is a cornerstone of flexible behavior that allows organisms to generalize existing knowledge to novel stimuli and to discriminate between physically similar, yet conceptually different, stimuli. Many real-world categories are defined by a combination of lowlevel visual properties, such as hue, luminance, spatial frequency, and orientation. For example, a forager might be tasked with determining whether a food source is edible based on subtle vari-

Received Nov. 14, 2019; accepted Dec. 3, 2019.

Author contributions: E.F.E. and J.T.S. designed research; E.F.E. and T.C.S. performed research; E.F.E. and T.C.S. analyzed data; E.F.E. wrote the first draft of the paper; E.F.E. wrote the paper; T.C.S. and J.T.S. edited the paper.

This work was supported by National Institutes of Health R01 EY025872 and a James S. McDonnell Foundation Award to J.T.S. We thank Kelvin Lam for assistance with data collection for Experiment 2.

The authors declare no competing financial interests.

Correspondence should be addressed to Edward F. Ester at eester@fau.edu.

https://doi.org/10.1523/JNEUROSCI.2700-19.2019

Copyright $\odot 2020$ the authors ations in color, shape, size, and texture. Humans and other animals can readily learn arbitrary novel categories defined by low-level visual properties (Goldstone, 1998; Ashby and Maddox, 2005), and such learning "distorts" perceptual sensitivity for category-defining features such that discrimination performance for physically similar yet categorically different stimuli is increased (i.e., acquired distinctiveness) (Goldstone, 1994; Newell and Bülthoff, 2002) and discrimination performance for stimuli from the same category reduced (i.e., acquired similarity) (Livingston et al., 1998).

Invasive electrophysiological studies suggest that single-unit responses in early visual areas index the physical properties of a stimulus but not its category membership, whereas single-unit responses in later areas index the category membership of a stimulus regardless of its physical properties (Freedman et al., 2001; e.g., Sigala and Logothetis, 2002; Freedman and Assad, 2006). These results have been taken as evidence that category-selective responses are a de novo property of higher-order visual areas. However, perceptual distortions following category learning 
could also reflect subtle changes in how to-be-categorized information is represented by sensory neural populations (Folstein et al., 2013; Davis and Poldrack, 2014). Here we provide a test of this possibility. In three experiments, we trained human participants (both sexes) to classify sets of continuous stimuli into discrete groups. Next, we applied multivariate models to noninvasive measurements of human brain activity (fMRI and EEG) from visual and parietal cortical areas while participants categorized the same stimulus sets. This allowed us to recover, visualize, and quantify stimulus-specific representations of to-becategorized exemplars. In Experiment 1 (fMRI), we show that reconstructed representations of to-be-categorized orientations in visual areas V1-V3 are systematically biased toward the center of the category to which they belong. These biases were correlated with trial-by-trial variability in overt category judgments and were largest for orientations adjacent to the category boundary where they would be most beneficial for discrimination performance. In Experiment 2, we used EEG to generate time-resolved representations of to-be-categorized orientations and show that categorical biases manifest shortly after stimulus onset $(\leq 300$ $\mathrm{ms}$ ). In Experiment 3, we used EEG and a delayed match-tocategory (DMC) task to show that categorical biases observed in Experiments 1 and 2 cannot be explained by response biases or motor preparation. Collectively, our findings suggest that mechanisms of categorization can shape information processing at the earliest stages of the visual system.

\section{Materials and Methods}

\section{General overview}

Participants. A total of 44 human volunteers (both sexes) participated in this study. Eight participants completed Experiment 1 (fMRI), 28 participants completed Experiment 2 (EEG), and 8 participants completed Experiment 3 (EEG). Experiments 1 and 2 were performed at the University of California, San Diego, while Experiment 3 was performed at Florida Atlantic University. Participants were recruited from the student body at each university. All study procedures were approved by local institutional review boards, and all participants gave both written and oral informed consent. Participants self-reported normal or correctedto-normal visual acuity and were remunerated with cash incentives ( $\$ 20 / \mathrm{h}$ for fMRI and $\$ 15 / \mathrm{h}$ for EEG).

Stimulus displays. Stimulus displays were generated in MATLAB and rendered using Psychophysics Toolbox software extensions (Kleiner et al., 2007). During Experiment 1 (fMRI), displays were projected onto a $110-\mathrm{cm}$-wide screen placed at the base of the MRI table, and participants viewed displays via a mirror attached to the MR head coil from a distance of $370 \mathrm{~cm}$. During Experiments 2 and 3, displays were projected onto a 19-inch CRT monitor cycling at $120 \mathrm{~Hz}$ (Experiment 2) or $85 \mathrm{~Hz}$ (Experiment 3). Participants were seated $\sim 65 \mathrm{~cm}$ from the display (head position was not constrained).

\section{Experiment 1: fMRI}

Participants. Eight neurologically intact human volunteers (AA, AB, AC, $\mathrm{AD}, \mathrm{AE}, \mathrm{AF}, \mathrm{AG}$, and $\mathrm{AH} ; 6$ females) completed Experiment 1. Each participant completed a single $1 \mathrm{~h}$ behavioral training session $\sim 24-72 \mathrm{~h}$ before scanning. Seven participants (AA, AB, AC, AD, AE, AF, AG) completed two $2 \mathrm{~h}$ experimental scan sessions; an eighth participant $(\mathrm{AH})$ completed a single $2 \mathrm{~h}$ experimental scan session. Participants $\mathrm{AA}, \mathrm{AB}$, $\mathrm{AC}, \mathrm{AD}, \mathrm{AE}, \mathrm{AF}$, and $\mathrm{AH}$ also completed a single $2 \mathrm{~h}$ retinotopic mapping scan session. Data from this session were used to identify visual field borders in early visual cortical areas $\mathrm{V} 1-\mathrm{hV} 4 / \mathrm{V} 3 \mathrm{~A}$ and subregions of posterior intraparietal sulcus (IPS0-3; see Retinotopic mapping).

Behavioral tasks. In separate runs (where "run" refers to a continuous block of 30 trials lasting 280 s), participants performed either an orientation mapping task or a category discrimination task. Trials in each task lasted $3 \mathrm{~s}$, and consecutive trials were separated by a 5 or $7 \mathrm{~s}$ intertrial interval (pseudorandomly chosen on each trial). During the orientation mapping task, participants attended a stream of letters presented at fixation (subtending $1.0^{\circ} \times 1.0^{\circ}$ from a viewing distance of $370 \mathrm{~cm}$ ) while ignoring a task-irrelevant phase-reversing $(15 \mathrm{~Hz})$ square-wave grating ( 0.8 cycles/deg with inner and outer radii of $1.16^{\circ}$ and $4.58^{\circ}$, respectively) presented in the periphery. On each trial, the grating was assigned 1 of 15 possible orientations $\left(0^{\circ}-168^{\circ}\right.$ in $12^{\circ}$ increments). Participants were instructed to detect and report the identity of a target (" $\mathrm{X}$ " or " $Y$ ") in the letter stream using an MR-compatible button box. Only one target was presented on each trial. Letters were presented at a rate of $10 \mathrm{~Hz}(50 \%$ duty cycle, i.e., $50 \mathrm{~ms}$ on, $50 \mathrm{~ms}$ off), and targets could occur during any cycle from 750 to $2250 \mathrm{~ms}$ after stimulus onset. During category discrimination runs, participants were shown displays containing a circular aperture (inner and outer radii of $1.16^{\circ}$ and $4.58^{\circ}$ from a viewing distance of $370 \mathrm{~cm}$ ) filled with 150 iso-oriented bars (see Fig. 1A). Each bar subtended $0.2^{\circ} \times 0.6^{\circ}$ with a stroke width of 8 pixels $(1024 \times 768$ display resolution). Each bar flickered at $30 \mathrm{~Hz}$ and was randomly replotted within the aperture at the beginning of each "up" cycle.

On each trial, all bars were assigned an orientation from $0^{\circ}$ to $168^{\circ}$ in $12^{\circ}$ increments. Inspired by earlier work in nonhuman primates (Freedman and Assad, 2006), we randomly selected and designated one of these orientations as a category boundary such that the seven orientations counterclockwise to this value were assigned membership in Category 1 , whereas the seven orientations clockwise to this value were assigned membership in Category 2. Participants were not informed that the category boundary was chosen from the set of possible stimulus orientations. Participants reported whether the orientation shown on each trial was a member of Category 1 or 2 (via an MR-compatible button box). Participants were free to respond at any point during the trial, although the stimulus was always presented for a total of $3000 \mathrm{~ms}$. Each participant was familiarized and trained to criterion performance on the category discrimination task during a $1 \mathrm{~h}$ behavioral testing session completed $1-3$ $\mathrm{d}$ before his or her first scan session. Written feedback ("Correct!" or "Incorrect") was presented in the center of the display for $1.25 \mathrm{~s}$ after each trial during behavioral training and MR scanning. Across either $1(N=$ $1)$ or $2(N=7)$ scan sessions, each participant completed $7(N=1), 13$ $(N=1), 14(N=1), 15(N=1)$, or $16(N=4)$ runs of the orientation mapping and category discrimination tasks.

$f M R I$ acquisition and preprocessing. Imaging data were acquired with a 3.0T GE MR 750 scanner located at the Center for Functional Magnetic Resonance imaging on the University of California, San Diego campus. All images were acquired with a 32 channel Nova Medical head coil. Whole-brain EPIs were acquired in $353 \mathrm{~mm}$ slices (no gap) with an in-plane resolution of $3 \times 3 \mathrm{~mm}(192 \times 192 \mathrm{~mm}$ FOV, $64 \times 64 \mathrm{~mm}$ image matrix, $90^{\circ}$ flip angle, $2000 \mathrm{~ms}$ TR, $30 \mathrm{~ms}$ TE). During retinotopic mapping scans (see below), EPIs were acquired in 31 3-mm-thick oblique slices (no gap) positioned over posterior visual and parietal cortex with an in-plane resolution of $2 \times 2 \mathrm{~mm}(192 \times 192 \mathrm{~mm}$ FOV, $96 \times$ $96 \mathrm{~mm}$ image matrix, $90^{\circ}$ flip angle, $2250 \mathrm{~ms}$ TR, $30 \mathrm{~ms} \mathrm{TE}$ ). EPIs were coregistered to a high-resolution anatomical image collected during the same session (FSPGR T1-weighted sequence, $11 \mathrm{~ms}$ TR, $3.3 \mathrm{~ms}$ TE, 1100 $\mathrm{ms}$ TI, 172 slices, $18^{\circ}$ flip angle, $1 \mathrm{~mm}^{3}$ resolution), unwarped (FSL software extensions), slice-time-corrected, motion-corrected, high-passfiltered (to remove first-, second-, and third-order drift), transformed to Talairach space, and normalized ( $z$ score) on a scan-by-scan basis. Data from data from scan sessions were then coregistered to a high-resolution anatomical image (FSPGR T1-weighted sequences; parameters as described above) collected during the retinotopic mapping session.

Retinotopic mapping. Retinotopically organized visual areas $\mathrm{V} 1-\mathrm{hV} 4 \mathrm{v} /$ V3A were defined using data from a single retinotopic mapping run collected during each experimental scan session. Participants fixated a small dot at fixation while phase-reversing $(8 \mathrm{~Hz})$ checkerboard wedges subtending $60^{\circ}$ of polar angle (at maximum eccentricity) were presented along the horizontal or vertical meridian (alternating with a period of $40 \mathrm{~s}$; i.e., $20 \mathrm{~s}$ of horizontal stimulation followed by $20 \mathrm{~s}$ of vertical stimulation). To identify visual field borders, we constructed a GLM with two boxcar regressors: one marking epochs of vertical stimulation and another marking epochs of horizontal stimulation. Each regressor was convolved with a canonical hemodynamic function ("double gamma" as implemented in BrainVoyager QX). Next, we generated a statistical para- 
metric map marking voxels with larger responses during epochs of vertical relative to horizontal stimulation. This map was projected onto a computationally inflated representation of each participant's cortical surface for visualization to aid in the definition of the borders of visual areas V1, V2v, V2d, V3v, V3d, hV4v, and V3A. Data from V2v and V2d were combined into a single V2 ROI, and data from V3v and V3d were combined into a single V3 ROI. ROIs were also combined across cortical hemispheres (e.g., left and right V1) as no asymmetries were observed and the stimulus was presented in the center of the visual field.

Seven participants (AA, AB, AC, AD, AE, AF, and $\mathrm{AH}$ ) completed a separate $2 \mathrm{~h}$ retinotopic mapping scan; data from this session were used to identify retinotopically organized regions of IPS0 -3. During each task run, participants were shown displays containing a rotating wedge stimulus (period 24.75 or $36 \mathrm{~s}$ ) that subtended $72^{\circ}$ of polar angle with inner and outer radii of $1.75^{\circ}$ and $8.75^{\circ}$, respectively. In alternating blocks, the wedge contained a $4 \mathrm{~Hz}$ phase-reversing checkerboard or field of moving dots, and participants were required to detect small, brief, and temporally unpredictable changes in checkerboard contrast or dot speed. Six participants completed between 8 and 14 task runs. To compute the best polar angle for each voxel in IPS, we shifted the signals from counterclockwise runs by twice the estimated $\mathrm{HRF}$ delay $(2 \times 6.75 \mathrm{~s}=13.5 \mathrm{~s})$, removed data from the first and last full stimulus cycle, and reversed the time series so that all runs reflected clockwise rotation. We next computed the power and phase of the response at the stimulus' period (either $1 / 24.75$ or $1 / 36$ $\mathrm{Hz})$ and subtracted the estimated HRF delay (6.75 s) to align the signal phase in each voxel with the stimulus' location. Maps of orientation preference (computed via cross-correlation) were projected onto a computationally inflated representation of each participant's gray-white matter boundary to aide in the identification of visual field borders separating IPSO -3. An eighth participant (AG) chose not to participate in an additional retinotopic mapping session. For this participant, we estimated visual field borders for visual areas V1-hV4/V3A using data from the retinotopic mapping run collected during the participant's sole experimental session. We did not attempt to define IPS regions IPS $0-3$ for this participant.

Decoding categorical biases in visual cortex. We used a linear decoder to examine whether fMRI activation patterns evoked by exemplars adjacent to the category boundary and at the center of each category were more similar during the category discrimination task relative to the orientation mapping task (i.e., acquired similarity). In the first phase of the analysis, we trained a linear support vector machine (LIBSVM implementation) (Chang and Lin, 2011) to discriminate between the oriented exemplars at the center of each category ( $48^{\circ}$ from the boundary) using data from the orientation mapping and category discrimination tasks. To ensure internal reliability, we implemented a "leave-one-run-out" cross validation scheme where data from all but one scanning run were used to train the classifier and data from the remaining scanning run were used for validation. This procedure was repeated until data from each scan had served as the validation set, and the results were averaged across permutations. Next, we trained a second classifier on activation patterns evoked by exemplars at the center of each category boundary and used the trained classifier to predict the category membership of exemplars adjacent to the category boundary. If category learning increases the similarity of activation patterns evoked by exemplars within the same category, then within-category decoding performance should be superior during the category discrimination task relative to the orientation mapping task.

Inverted encoding model of orientation selectivity. A linear inverted encoding model was used to recover a model-based representation of stimulus orientation from multivoxel activation patterns measured in early visual areas (Brouwer and Heeger, 2011). The same general approach was used during Experiments 1 (fMRI) and 2 (EEG). Specifically, we modeled the responses of voxels (electrodes) measured during the orientation mapping task as a weighted sum of 15 orientation-selective channels, each with an idealized response function (half-wave-rectified sinusoid raised to the 14th power). The maximum response of each channel was set to unit amplitude; thus, units of response are arbitrary. Let $B_{1}(m$ voxels or electrodes $\times n_{1}$ trials) be the response of each voxel (electrode) during each trial of the RSVP task, let $C_{1}$ ( $k$ filters $\times n_{1}$ trials) be a matrix of hypothetical orientation filters, and let $W$ ( $m$ voxels or electrodes $\times k$ filters) be a weight matrix describing the mapping between $B_{1}$ and $C_{1}$ as follows:

$$
B_{1}=W C_{1}
$$

In the first phase of the analysis, we computed the weight matrix $W$ from the voxelwise (electrodewise) responses in $B_{1}$ via ordinary least squares as follows:

$$
W=B_{1} C_{1}^{T}\left(C_{1} C_{1}^{T}\right)^{-1}
$$

Next, we defined a test dataset $B_{2}$ ( $m$ voxels or electrodes $\times n_{2}$ trials) using data from the category discrimination task. Given $W$ and $B_{2}$, a matrix of filter responses $C_{2}$ ( $k$ filters $\times n$ trials) can be estimated via model inversion as follows:

$$
C_{2}=\left(W^{T} W\right)^{-1} W^{T} B_{2}
$$

$C_{2}$ contains the reconstructed response of each modeled orientation channel (the channel response function [CRF]) on each trial of the category discrimination task. This analysis can be considered a form of model-based, directed dimensionality reduction where activity patterns are transformed from their original measurement space (fMRI voxels; EEG electrodes) into a modeled information space (orientation-selective channels). Importantly, results from this method cannot be used to infer any changes in orientation tuning, or any properties of neural responses, occurring at the single-neuron level, and only assay the information content of large-scale patterns of neural activity (Sprague et al., 2018). Additionally, while it is the case that arbitrary linear transforms can be applied to the basis set, model weights, and reconstructed CRF (Gardner and Liu, 2019), results are uniquely defined for a given model specification (Sprague et al., 2018). Trial-by-trial CRFs were multiplied by the original basis set to recover a full $180^{\circ}$ function, circularly shifted to a common center $\left(0^{\circ}\right)$, and sorted by category membership so that any category bias would manifest as a clockwise shift (i.e., toward the center of Category 2).

Quantification of bias in orientation representations. To quantify categorical biases in reconstructed model-based CRFs, these functions were fit with an exponentiated cosine function of the following form:

$$
f(x)=\alpha\left(e^{k(\cos (\mu-x)-1)}\right)+\beta
$$

where $x$ is a vector of channel responses and $\alpha, \beta, k$, and $\mu$ correspond to the amplitude (i.e., signal over baseline), baseline, concentration (the inverse of bandwidth), and the center of the function, respectively. Fitting was performed using a multidimensional nonlinear minimization algorithm (Nelder-Mead).

Category biases in the estimated center of each construction $(\mu)$ during the category discrimination task were quantified via permutation tests. For a given visual area (e.g., V1), we randomly selected (with replacement) stimulus reconstructions from 8 of 8 participants. Specifically, we computed a "mean" reconstruction by randomly selecting (with replacement) and averaging reconstructions from all participants. The mean reconstruction was fit with the cosine function described above, yielding point estimates of $\alpha, \beta, k$, and $\mu$. This procedure was repeated 1000 times, yielding 1000 element distributions of parameter estimates. We then computed the proportion of permutations where a $\mu$ value $<0$ to obtain an empirical $p$ value for categorical shifts in reconstructed representations.

Searchlight decoding of category membership. We used a roving searchlight analysis (Ester et al., 2015) to identify cortical regions beyond V1-V3 that contained category-specific information. We defined a spherical neighborhood with a radius of $8 \mathrm{~mm}$ around each gray matter voxel in the cortical sheet. We next extracted and averaged the normalized response of each voxel in each neighborhood over a period from 4 to $8 \mathrm{~s}$ after stimulus onset (this interval was chosen to account for typical hemodynamic lag of 4-6 s). A linear support vector machine (LIBSVM implementation) was used to classify stimulus category using activation patterns within each neighborhood. To classify category membership, we designated the three orientations immediately counterclockwise to the category boundary (see Fig. 1) as members of Category 1 and the three 
orientations immediately clockwise of the boundary as members of Category 2 . We then trained our classifier to discriminate between categories using data from all but one task run. The trained classifier was then used to predict category membership from activation patterns measured during the held-out task run. This procedure was repeated until each task run had been held out, and the results were averaged across permutations. Finally, we repeated the same analysis using the three Category 1 and Category 2 orientations adjacent to the second (orthogonal) category boundary (see Fig. 1) and averaged the results across category boundaries.

We identified neighborhoods encoding stimulus category using a leave-one-participant-out cross validation approach (Esterman et al., 2010). Specifically, for each participant (e.g., AA), we randomly selected (with replacement) and averaged classifier performance estimates from each neighborhood from each of the remaining 7 volunteers (e.g., AB$\mathrm{AH})$. This procedure was repeated 1000 times, yielding a set of 1000 classifier performance estimates for each neighborhood. We generated a statistical parametric map for the held-out participant that indexed neighborhoods where classifier performance was greater than chance (50\%) on $97.5 \%$ of permutations (false-discovery-rate corrected for multiple comparisons across neighborhoods). Finally, we projected each participant's statistical parametric map onto a computationally inflated representation of his or her gray-white matter boundary and used Brain Voyager's "Create POIs from Map Clusters" function with an area threshold of $25 \mathrm{~mm}^{2}$ to identify ROIs supporting above-chance category classification performance. Because of differences in cortical folding patterns, some ROIs could not be unambiguously identified in all 8 participants. Therefore, across participants, we retained all ROIs that were shared by at least 7 of 8 participants. Finally, we extracted multivoxel activation patterns from each ROI and computed model-based reconstructions of CRFs during the RSVP and category tasks using a leave-onerun-out cross-validation approach. Specifically, we used data from all but one task run to estimate a set of orientation weights for each voxel in each ROI. We then used these weights and activation patterns measured during the held-out task run to estimate a CRF, which contains a representation of stimulus orientation. This procedure was repeated until each task run had been held out, and the results were averaged across permutations. Each participant's ROIs were defined using data from the remaining 7 participants. This ensured that participant-level reconstructions were statistically independent of the searchlight method used to define ROIs encoding category information.

Within-participant error bars. We report estimates of within-participant variability (e.g., \pm 1 SEM) throughout the paper. These estimates discard subject variance (e.g., overall differences in BOLD response amplitude) and instead reflect variance related to the subject by condition(s) interaction term(s) (i.e., variability in estimated channel responses). We used the approach described by Cousineau (2005): raw data (e.g., channel response estimates) were de-meaned on a participant-by-participant basis, and the grand mean across participants was added to each participant's zero-centered data. The grand mean-centered data were then used to compute estimates of SE.

\section{Experiment 2: EEG}

Participants. Twenty-nine new volunteers recruited from the University of California, San Diego community completed Experiment 2. All participants self-reported normal or corrected-to-normal visual acuity and gave both written and oral informed consent as required by the local Institutional Review Board. Each participant was tested in a single $2.5-3 \mathrm{~h}$ experimental session (the exact duration varied across participants depending on the amount of time needed to set up and calibrate the EEG equipment). Unlike Experiment 1, participants were not trained on the categorization task before testing. We adopted this approach in the hopes of tracking the gradual emergence of categorical biases during learning. However, many participants learned the task relatively quickly (within 40-60 trials), leaving too few trials to enable a direct analysis of this possibility. Data from 1 participant were discarded due to a high number of EOG artifacts ( $>35 \%$ of trials); the data reported here reflect the remaining 28 participants.
Behavioral tasks. In separate runs (where "run" refers to a continuous block of 60 trials lasting $\sim 6.5 \mathrm{~min}$ ), participants performed orientation mapping and category discrimination tasks similar to those used in Experiment 1 . During both tasks, a rapid series of letters (subtending $1.14^{\circ} \times$ $1.14^{\circ}$ from a viewing distance of $55 \mathrm{~cm}$ ) was presented at fixation, and an aperture of 150 iso-oriented bars (subtending $0.5^{\circ} \times 1.2^{\circ}$ ) was presented in the periphery. The aperture of bars had inner and outer radii of $1.96^{\circ}$ and $9.13^{\circ}$, respectively. On each trial, the bars were assigned 1 of 15 possible orientations (again $0^{\circ}-168^{\circ}$ in $12^{\circ}$ increments) and flickered at a rate of $30 \mathrm{~Hz}$. Each bar was randomly replotted within the aperture at the beginning of each "up" cycle. Letters in the RSVP stream were presented at a rate of $6.67 \mathrm{~Hz}$.

During orientation mapping runs, participants detected and reported the presence of a target letter (an X or Y) that appeared at an unpredictable time during the interval from 750 to $2250 \mathrm{~ms}$ following stimulus onset. Responses were made on a USB-compatible number pad. During category discrimination runs, participants ignored the RSVP stream and instead reported whether the orientation of the bar aperture was an exemplar from Category 1 or Category 2. As in Experiment 1, we randomly designated 1 of the 15 possible stimulus orientations as the category boundary such that the seven orientations counterclockwise to this value were assigned to Category 1 and the seven orientations clockwise to this value were assigned to Category 2. Participants could respond at any point during the trial, but the stimulus was presented for a total of 3000 ms. Trials were separated by a $2.5,3.25 \mathrm{~s}$ intertrial interval (randomly selected from a uniform distribution on each trial). Each participant completed $4(N=1), 5(N=10), 6(N=8), 7(N=8)$, or $8(N=1)$ blocks of the category task and $3(N=1), 4(N=1), 5(N=5), 6(N=$ $12), 7(N=8)$, or $8(N=1)$ blocks of the orientation mapping task.

EEG acquisition and preprocessing. Participants were seated in a dimly lit, sound-attenuated, and electrically shielded recording chamber (ETSLindgren) for the duration of the experiment. Continuous EEG was re-

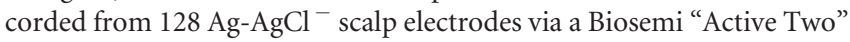
system. The horizontal EOG was recorded from additional electrodes placed near the left and right canthi, and the vertical EOG was recorded from electrodes placed above and below the right eye. Additional electrodes were placed over the left and right mastoids. The horizontal and vertical EOGs were recorded from electrodes placed over the left and right canthi and above and below the right eye, respectively. Electrode impedances were kept well below $20 \mathrm{k} \Omega$, and recordings were digitized at $1024 \mathrm{~Hz}$.

After testing, the entire EEG time series at each electrode was high- and low-pass filtered (third-order zero-phase forward and reverse Butterworth) at 0.1 and $50 \mathrm{~Hz}$ and rereferenced to the average of the left and right mastoids. Data from both tasks were epoched into intervals spanning -1000 to $4000 \mathrm{~ms}$ from stimulus onset; the relatively large prestimulus and poststimulus epochs were included to absorb filtering artifacts that could affect later analyses. Trials contaminated by EOG artifacts (horizontal eye movements $>2^{\circ}$ and blinks) were identified and excluded from additional analyses. Across participants an average of $5.58 \%( \pm 1.67 \%)$ and $8.74 \%( \pm 1.84 \%)$ of trials from the orientation mapping and category discrimination tasks were discarded, respectively. Finally, noisy channels (those with multiple deflections $\geq 100 \mu \mathrm{V}$ over the course of the experiment) were visually identified and eliminated (mean number of removed electrodes across participants \pm 1 SEM: $2.25 \pm 0.64)$.

Next, we identified a set of electrodes of interest with strong responses at the stimulus' flicker frequency $(30 \mathrm{~Hz})$. Data from each task were re-epoched into intervals spanning 0 to $3000 \mathrm{~ms}$ around stimulus onset and averaged across trials and tasks (i.e., RSVP and category discrimination), yielding a $k$ electrode by $t$ sample data matrix. We computed the evoked power at the stimulus' flicker frequency $(30 \mathrm{~Hz})$ by applying a discrete Fourier transform to the average time series at each electrode and selected the 32 electrodes with the highest evoked power at the stimulus' flicker frequency for further analysis. These electrodes were typically distributed over occipitoparietal electrode sites (see Fig. 12).

To isolate stimulus-specific responses, the epoched time series at each electrode was resampled to $256 \mathrm{~Hz}$ and then bandpass filtered from 29 to $31 \mathrm{~Hz}$ (zero-phase forward and reverse third-order Butterworth). We 
next estimated a set of complex Fourier coefficients describing the power and phase of the $30 \mathrm{~Hz}$ response by applying a Hilbert transformation to the filtered data. To visualize and quantify orientation-selective signals from frequency-specific responses, we first constructed a complex valued dataset $B_{1}(t)$ ( $m$ electrodes $\times n_{\text {train }}$ trials). We then estimated a complex valued weight matrix $W(t)$ ( $m$ channels $\times k$ filters) using $B_{1}(t)$ and a basis set of idealized orientation-selective filters $C_{1}$. Finally, we estimated a complex valued matrix of channel responses $C_{2}(t)\left(m\right.$ channels $\times n_{\text {test }}$ trials) given $W(t)$ and complex valued test dataset $B_{2}(t)$ ( $m$ electrodes $\times$ $n_{\text {test }}$ trials) containing the complex Fourier coefficients measured during the category discrimination task. Trial-by-trial and sample-by-sample response functions were shifted in the same manner described above so that category biases would manifest as a rightward (clockwise) shift toward the center of Category 2. We estimated the evoked (i.e., phaselocked) power of the response at each filter by computing the squared absolute value of the average complex valued coefficient for each filter after shifting. Categorical biases were quantified using the same curve fitting analysis described in the main text.

To obtain an unbiased estimate of orientation selectivity in each electrode, we ensured that the training dataset $B_{1}(t)$ contained an equal number of trials for each stimulus orientation $\left(0^{\circ}-168^{\circ}\right.$ in $12^{\circ}$ increments). For each participant, we identified the stimulus orientation $\theta$ with the $N$ fewest repetitions in the orientation mapping dataset after EOG artifact removal. Next, we constructed the training dataset $B_{1}(t)$ by randomly selecting (without replacement) 1:N trials for each stimulus orientation. Data from this training set were used to estimate a set of orientation weights for each electrode, and these weights were in turn used to estimate a response for each hypothetical orientation channel during the category discrimination task. To ensure that our method generalized across multiple combinations of orientation mapping trials, we repeated this analysis 100 times and averaged the results across permutations.

\section{Experiment 3: EEG}

Participants. Eight volunteers recruited from the Florida Atlantic University community completed Experiment 3 . All participants self-reported normal or corrected-to-normal visual acuity and gave both written and oral informed consent as required by the local Institutional Review Board. Each participant was tested in a single $2-2.5 \mathrm{~h}$ experimental session (the exact duration varied across participants depending on the amount of time needed to set up and calibrate the EEG equipment).

Behavioral tasks. Participants performed six blocks of a spatial recall task followed by multiple blocks of a DMC task. Both tasks used identical stimulus and display geometry. During the spatial recall task, participants were shown a sample display containing a disc (diameter $2.5^{\circ}$ from a viewing distance of $60 \mathrm{~cm})$ rendered in 1 of 12 polar locations $\left(0^{\circ}-330^{\circ}\right.$ in $30^{\circ}$ increments) along the perimeter of an imaginary circle centered at fixation (radius $7.5^{\circ}$ ). The sample display was shown for $250 \mathrm{~ms}$ and followed by a $1750 \mathrm{~ms}$ blank delay. At the end of each trial, participants were shown a mouse cursor and instructed to click on the position of the disc shown in the sample display. Participants were instructed to prioritize accuracy over speed, although a $3000 \mathrm{~ms}$ response deadline was imposed. Each trial was followed by a 1500-2200 ms blank interval (randomly sampled from a uniform distribution on each trial). Each block featured 72 trials (six repetitions per stimulus position) and lasted $\sim 6$ min. EEG data recorded during this task were used to train a positionspecific inverted encoding model (see below). Each participant completed six blocks of this task.

After completing the spatial recall task, participants performed a DMC task. Participants were shown stimuli in the same 12 positions used during the spatial recall task. However, for each participant, we defined a category boundary such that half of the possible stimulus positions were assigned membership in Category 1 and the remaining half were assigned membership in Category 2. For example, the category boundary could be set such that positions $[315,345,15,45,75,105]$ comprised Category 1 , whereas positions $[135,165,195,225,255,285]$ comprised Category 2. The location of the category boundary was randomly and independently chosen for each participant and held constant throughout the experiment. At the beginning of each trial, a sample disc appeared in 1 of the 12 possible stimulus locations for $250 \mathrm{~ms}$. After a $1750 \mathrm{~ms}$ delay period, a probe disc was presented. The probe could occupy any of the 11 stimulus positions not occupied by the sample, and participants were required to judge whether the position of the probe matched the category of the sample stimulus via keypress. Participants were instructed to prioritize accuracy over speed, but a $3000 \mathrm{~ms}$ response limit was imposed. Feedback (correct vs incorrect) was presented at the end of each trial. Participants completed $5(N=1)$ or $8(N=7)$ blocks of 72 trials.

EEG acquisition and preprocessing. Continuous EEG was recorded from $63 \mathrm{Ag} /{\mathrm{Ag}-\mathrm{Cl}^{-}}^{-}$scalp electrodes via a Brain Products actiCHamp amplifier. An additional electrode was placed over the right mastoid. Data were recorded with a right mastoid reference and later rereferenced to the algebraic mean of the left and right mastoids (10-20 site TP9 served as the left mastoid reference). The horizontal and vertical EOG was recorded from electrodes placed on the left and right canthi and above and below the right eye, respectively. All electrode impedances were kept to $<15 \mathrm{k} \Omega$, and recordings were digitized at $1000 \mathrm{~Hz}$. Recorded data were bandpass filtered from 1 to $50 \mathrm{~Hz}$ (third-order zero-phase forward and reverse Butterworth filters), epoched from a period spanning -1000 to $3000 \mathrm{~ms}$ relative to the start of each trial, and baseline corrected from -250 to $0 \mathrm{~ms}$. Muscle and EOG artifacts were removed from the data using independent components analysis as implemented in EEGLAB (Delorme and Makeig, 2004). Reconstructions of stimulus locations were computed from the spatial topography of induced alphaband $(8-12 \mathrm{~Hz})$ power measured across 17 occipitoparietal electrode sites: O1, O2, Oz, PO7, PO3, POz, PO4, PO8, P7, P5, P3, P1, Pz, P2, P4, $\mathrm{P} 6$, and $\mathrm{P} 8$. Inverted encoding model. Experiment 3 relied on a fundamentally different signal than Experiment 2 (induced-alpha-band activity vs evoked $30 \mathrm{~Hz}$ power, respectively). Following earlier research (Kok et al., 2017; Ester et al., 2018; Nouri and Ester, 2020), we used a variant of the inverted encoding model approach described in Experiment 2 to compute location channel responses. We first isolated alpha-band activity, by bandpass filtering the raw EEG time series at each electrode from 8 to 12 $\mathrm{Hz}$ (zero-phase forward and reverse filters as implemented by EEGLAB's "eegfilt" function), yielding a real valued signal $f(t)$. The analytic representation of $f(t)$ was obtained by applying a Hilbert transformation as follows:

$$
z(t)=f(t)+i f(t)
$$

where $i=V-1$ and $i f(t)=A(t) e^{i \varphi(t)}$. Induced alpha power was computed by extracting and squaring the instantaneous amplitude $A(t)$ of the analytic signal $z(t)$. We modeled alpha power at each scalp electrode as a weighted sum of 12 location-selective channels, each with an idealized tuning curve (a half-wave rectified cosine raised to the 12th power). The maximum response of each channel was normalized to 1 ; thus, units of response are arbitrary. The predicted responses of each channel during each trial were arranged in a $k$ channel by $n$ trials design matrix $C$. Separate design matrices were constructed to track the locations of the blue and red discs across trials (i.e., we reconstructed the locations of the blue and red discs separately and then later sorted these reconstructions according to cue condition). The relationship between the data and the predicted channel responses $C$ is given by a GLM of the following form:

$$
B=W C+N
$$

where $B$ is an $m$ electrode by $n$ trials training data matrix, $W$ is an $m$ electrode by $k$ channel weight matrix, and $N$ is a matrix of residuals (i.e., noise).

To estimate $W$, we constructed a "training" dataset containing an equal number of trials from each stimulus location (i.e., $45-360^{\circ}$ in $45^{\circ}$ steps) condition. We first identified the location $\varphi$ with the fewest $r$ repetitions in the full dataset after EOG artifact removal. Next, we constructed a training dataset $B_{t r n}$ ( $m$ electrodes by $n$ trials) and weight matrix $C_{t r n}$ ( $n$ trials by $k$ channels) by randomly selecting (without replacement) $1: r$ trials for each of the eight possible stimulus locations (ignoring cue condition; i.e., the training dataset contained a mixture of neutral and valid trials). The training dataset was used to compute a weight for each channel $C_{i}$ via least-squares estimation as follows: 


$$
W_{i}=B_{t r n} C_{t r n, 1}^{T}\left(C_{t r n, i} C_{t r n, 1}^{T}\right)^{-1}
$$

where $C_{t r n, i}$ is an $n$ trial row vector containing the predicted responses of spatial channel $i$ during each training trial.

After estimating the weight matrix $W$, we next estimated a set of spatial filters $V$ that capture the underlying channel responses while accounting for correlated variability between electrode sites (i.e., the noise covariance) (Kok et al., 2017) as follows:

$$
V_{i}=\frac{\sum_{i}^{-1} W_{i}}{W_{i}^{T} \sum_{i}^{-1} W_{i}}
$$

where $\Sigma_{i}$ is the regularized noise covariance matrix for channel $i$ and estimated as follows:

$$
\sum_{i}=\frac{1}{n-1} \in_{i} \in_{i}^{T}
$$

where $n$ is the number of training trials and $\varepsilon_{i}$ is a matrix of residuals as follows:

$$
\epsilon_{i}=B_{t r n}-W_{i} C_{t r n, i}
$$

Estimates of $\varepsilon_{i}$ were obtained by regularization-based shrinkage using an analytically determined shrinkage parameter (see Blankertz et al., 2011; Kok et al., 2017). An optimal spatial filter $v_{i}$ was estimated for each channel $C_{i}$, yielding an $m$ electrode by $k$ filter matrix $V$. Next, we constructed a "test" dataset $B_{t s t}$ ( $m$ electrodes by $n$ trials) containing data from all trials not included in the training dataset and estimated trial-bytrial channel responses $C_{t s t}(k$ channels $\times n$ trials $)$ from the filter matrix $V$ and the test dataset as follows:

$$
C_{t s t}=V^{T} B_{t s t}
$$

Trial-by-trial channel responses were interpolated to $360^{\circ}$, circularly shifted to a common center $\left(0^{\circ}\right.$, by convention), and sorted by category membership. As in Experiments 1 and 2, reconstructions were shifted and aligned so that any bias would manifest as a shift toward Category B (clockwise). Finally, to ensure internal reliability, this entire analysis was repeated 50 times, and unique (randomly chosen) subsets of trials were used to define the training and test datasets during each permutation. The results were then averaged across permutations.

Eye movement control analyses, Experiments 2 and 3. Systematic biases in eye position can contribute to orientation and location performance (e.g., Quax et al., 2019). We did not collect eye position data from Experiment 1 (fMRI). However, different tasks were used to train and test the encoding model, which can be an effective way of mitigating the effects of eye movements on stimulus decoding (Mostert et al., 2018). We also collected EOG data during Experiments 2 and 3 (EEG). To examine whether eye position varied as a function of stimulus position during these experiments, we regressed trial-by-trial horizontal EOG recordings (in $\mu \mathrm{V}$ ) onto the orientation of a to-be-categorized stimulus (Experiment 2) or the location of a to-be-categorized disc (Experiment 3). In both experiments, we identified and excluded trials contaminated by large horizontal EOG artifacts ( $\geq 40 \mu \mathrm{V}$, which corresponds to a horizontal displacement of $2.5^{\circ}$ assuming a voltage threshold of $16 \mu \mathrm{V}$ per degree) (Lins et al., 1993), but smaller variations in eye positions, for example, along the inner stimulus aperture, may have escaped detection. Using Experiment 2 as an example, we considered two possibilities. First, participants may have foveated the inner aperture of the stimulus at a polar location matching its orientation. To illustrate, participants could foveate the inner aperture of a $45^{\circ}$ stimulus at a polar angle of $45^{\circ}$ or $225^{\circ}$; likewise, they could foveate the inner aperture of a $168^{\circ}$ stimulus at a polar angle of $168^{\circ}$ or $348^{\circ}$. Second, participants may have foveated the inner aperture of each stimulus matching the center of the category to which it belonged. We tested these possibilities by calculating predicted horizontal eye positions under the assumption that participants foveated the inner stimulus aperture at locations matching its orientation or the center of the relevant category. Specifically, we converted records of stimulus orientation (or the center of the category to which the stimulus
A

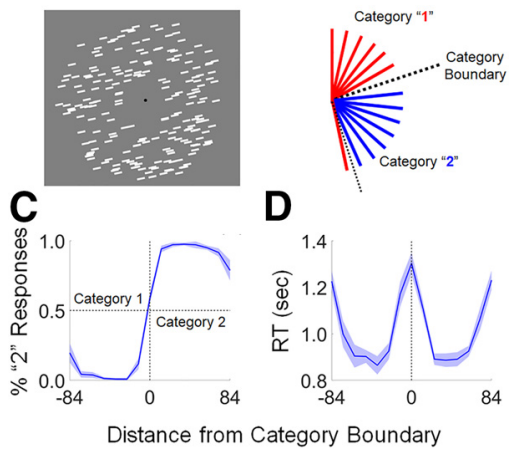

Figure 1. Overview of Experiment 1. A, Participants viewed displays containing a circular aperture of iso-oriented bars. On each trial, the bars were assigned 1 of 15 unique orientations from $0^{\circ}$ to $168^{\circ}$. B, We randomly selected and designated one stimulus orientation as a category boundary (black dashed line) such that the seven orientations counterclockwise from this value were assigned to Category 1 (red lines) and the seven orientations clockwise from this value were assigned to Category 2 (blue lines). C, After training, participants rarely miscategorized orientations. $\boldsymbol{D}$, Response latencies are significantly longer for oriented exemplars near the category boundary. RT, Response time. $C, D$, Shaded regions represent \pm 1 within-participant SEM.

belonged) to polar format and scaled the resulting estimates by the radius of the inner stimulus aperture, then regressed these estimates onto horizontal EOG activity (in $\mu \mathrm{V}$ ). If there is a systematic relationship between eye position and either stimulus orientation or category at any point during a trial, then this analysis should yield regression coefficients reliably $>0 \mu \mathrm{V}$. Identical analyses were used to examine systematic relationships between horizontal eye position and stimulus location in Experiment 3.

\section{Results}

\section{Experiment 1: fMRI}

We trained 8 human volunteers to categorize a set of orientations into two groups, Category 1 and Category 2. The stimulus space comprised a set of 15 oriented stimuli, spanning $0^{\circ}-168^{\circ}$ in $12^{\circ}$ increments (Fig. $1 A, B$ ). For each participant, we randomly designated 1 of these 15 orientations as a category boundary such that the seven orientations anticlockwise to the boundary were assigned membership in Category 1 and the seven orientations clockwise to the boundary were assigned membership in Category 2. Each participant completed a $1 \mathrm{~h}$ training session before scanning. Each participant's category boundary was kept constant across all behavioral training and scanning sessions. Many participants self-reported that they learned the rule delineating the categories in one or two 5 min blocks of trials. Consequently, task performance measured during scanning was extremely high, with errors and slow responses present only for exemplars immediately adjacent to the category boundary (Fig. $1 C, D$ ). During each scanning session, participants performed the category discrimination task and an orientation model estimation task where they were required to report the identity of a target letter embedded within a rapid stream presented at fixation while a taskirrelevant grating flickered in the background. Data from this task were used to compute an unbiased estimate of orientation selectivity for each voxel in visual areas $\mathrm{V} 1-\mathrm{hV} 4 \mathrm{v} / \mathrm{V} 3 \mathrm{~A}$ (see below).

We first examined whether category training increased the similarity of activation patterns evoked by exemplars from the same category (i.e., acquired similarity). We tested this by training a linear decoder (support vector machine) to discriminate between activation patterns associated with exemplars at the cen- 


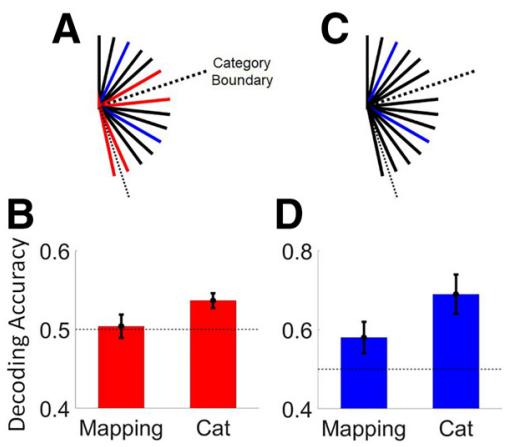

Figure 2. Category decoding performance. $A$, We trained classifiers on activation patterns evoked by exemplars at the center of each category boundary during the orientation mapping and category discrimination task (blue lines) and then used the trained classifier to predict the category membership of exemplars adjacent to the category boundary (red lines). B, Decoding accuracy was significantly higher during the category discrimination task relative to the orientation mapping task ( $p=0.01$ ), suggesting that activation patterns evoked by exemplars adjacent to the category boundary became more similar to activation patterns evoked by exemplars at the center of each category during the categorization task. The absence of robust decoding performance during the orientation mapping task cannot be attributed to poor signal or a uniform enhancement of orientation representations by attention, as a decoder trained and tested on activation patterns associated with exemplars at the center of each category $(\boldsymbol{C})$ yielded above-chance decoding during both behavioral tasks (D). Decoding performance was computed from activation patterns in V1. Error bars indicate \pm 1 SEM.

ter of each category ( $48^{\circ}$ from the boundary) and then used the trained classifier to predict the category membership of exemplars immediately adjacent to the category boundary $\left( \pm 12^{\circ}\right.$; Fig. $2 A)$. This analysis was performed separately for the orientation mapping and category discrimination tasks. We reasoned that, if category training homogenizes activation patterns evoked by members of the same category, then decoding performance should be superior during the category discrimination task relative to the orientation mapping task. This is precisely what we observed (Fig. 2B). For example, near-boundary decoding performance in V1 was reliably above chance during the category discrimination task ( $p<0.0001$, FDR-corrected bootstrap test), but not during the orientation mapping task when the category boundary was irrelevant and the oriented stimulus was unattended ( $p=0.38$ ). Importantly, the absence of robust decoding performance during the orientation mapping task cannot be attributed to poor signal, as a decoder trained and tested on activation patterns associated with exemplars at the center of each category (Fig. 2C) yielded above-chance decoding during both behavioral tasks (Fig. 2D; mean 0.58 and 0.69 for the mapping and discrimination tasks, respectively; $p<0.01$, bootstrap test). Collectively, these results suggest that category training can alter population-level responses at very early stages of the visual processing hierarchy.

To better understand how category training influences orientationselective activation patterns in early visual cortical areas, we used an inverted encoding model (Brouwer and Heeger, 2011) to generate model-based reconstructed representations of stimulus orientation from these patterns. For each visual area (e.g., V1), we first modeled voxelwise responses measured during the orientation mapping task as a weighted sum of idealized orientation channels, yielding a set of weights that characterize the orientation selectivity of each voxel (Fig. $3 A$ ). In the second phase of the analysis, we reconstructed trial-by-trial representations of stimulus orientation by combining these weights with the observed pattern of activation across voxels measured during each trial of the category discrimination task, resulting in single-trial re-
A

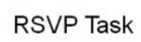

Voxel Responses

Basis Set
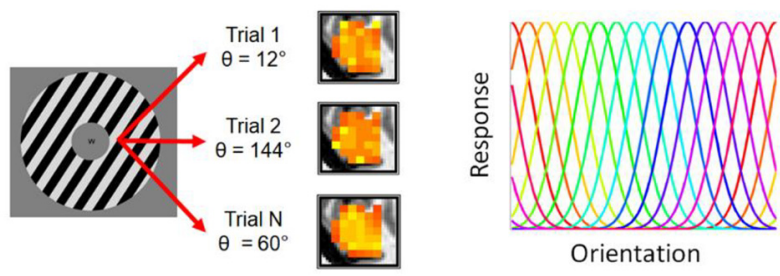

B

Voxel Weights

Stimulus Reconstruction
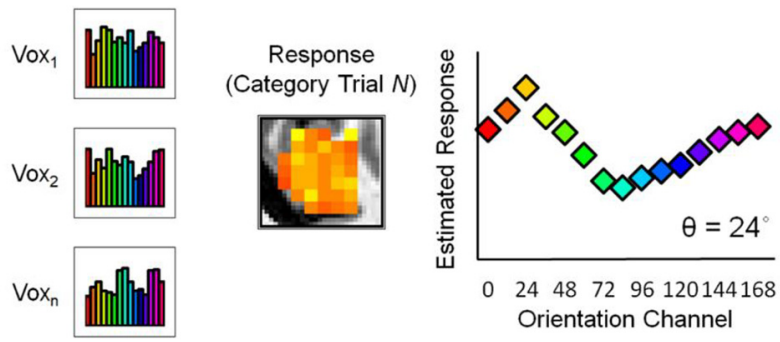

Figure 3. Inverted encoding model. $\boldsymbol{A}$, In the first phase of the analysis, we estimated an orientation selectivity profile for each voxel retinotopically organized V1-hV4/V3a using data from an independent orientation mapping task. Specifically, we modeled the response of each voxel as a set of 15 hypothetical orientation channels, each with an idealized response function. $\boldsymbol{B}$, In the second phase of the analysis, we computed the response of each orientation channel from the estimated orientation weights and the pattern of responses across voxels measured during each trial of the category discrimination task. The resulting reconstructed CRF contains a representation of the stimulus orientation (example; $24^{\circ}$ ), which we quantified via a curvefitting procedure.

constructed CRF that contains a representation of stimulus orientation for each ROI on each trial (Fig. 3B). Finally, we sorted trial-by-trial reconstructions according to category membership such that any bias would manifest as a clockwise (rightward) shift of the orientation representation toward the center of Category 2 and quantified biases toward this category using a curve-fitting analysis (see Materials and Methods).

Stimulus orientation was irrelevant during the orientation mapping task used for model weight estimation. We therefore reasoned that voxel-by-voxel responses evoked by each oriented stimulus would be largely uncontaminated by its category membership. Indeed, the logic of our analytical approach rests on the assumption that orientation-selective responses are quantitatively different during the orientation mapping and category discrimination tasks: if identical category biases are present in both tasks, then the orientation weights computed using data from either task will capture that bias and reconstructed representations of orientation will not exhibit any category shift. This is precisely what we observed when we used a cross-validation approach to reconstruct stimulus representations separately for the orientation mapping and category discrimination tasks (Fig. 4).

As shown in Figure 5, reconstructed representations of orientation in visual areas V1, V2, and V3 were systematically biased away from physical stimulus orientation and toward the center of the appropriate category (shifts of $22.13^{\circ}, 26.65^{\circ}$, and $34.57^{\circ}$, respectively; $p<0.05$, bootstrap test, false discovery rate [FDR] corrected for multiple comparisons across regions; see Fig. 6 for separate reconstructions of Category 1 and Category 2 orientations and Fig. 7 for participant-by-participant reconstructions plotted by visual area). Similar, though less robust, biases were also evident in $\mathrm{hV} 4 \mathrm{v}$ and $\mathrm{V} 3 \mathrm{~A}$ (mean shifts of $9.73^{\circ}$ and $6.45^{\circ}$, respectively; $p>0.19$ ). A logistic regression analysis established 


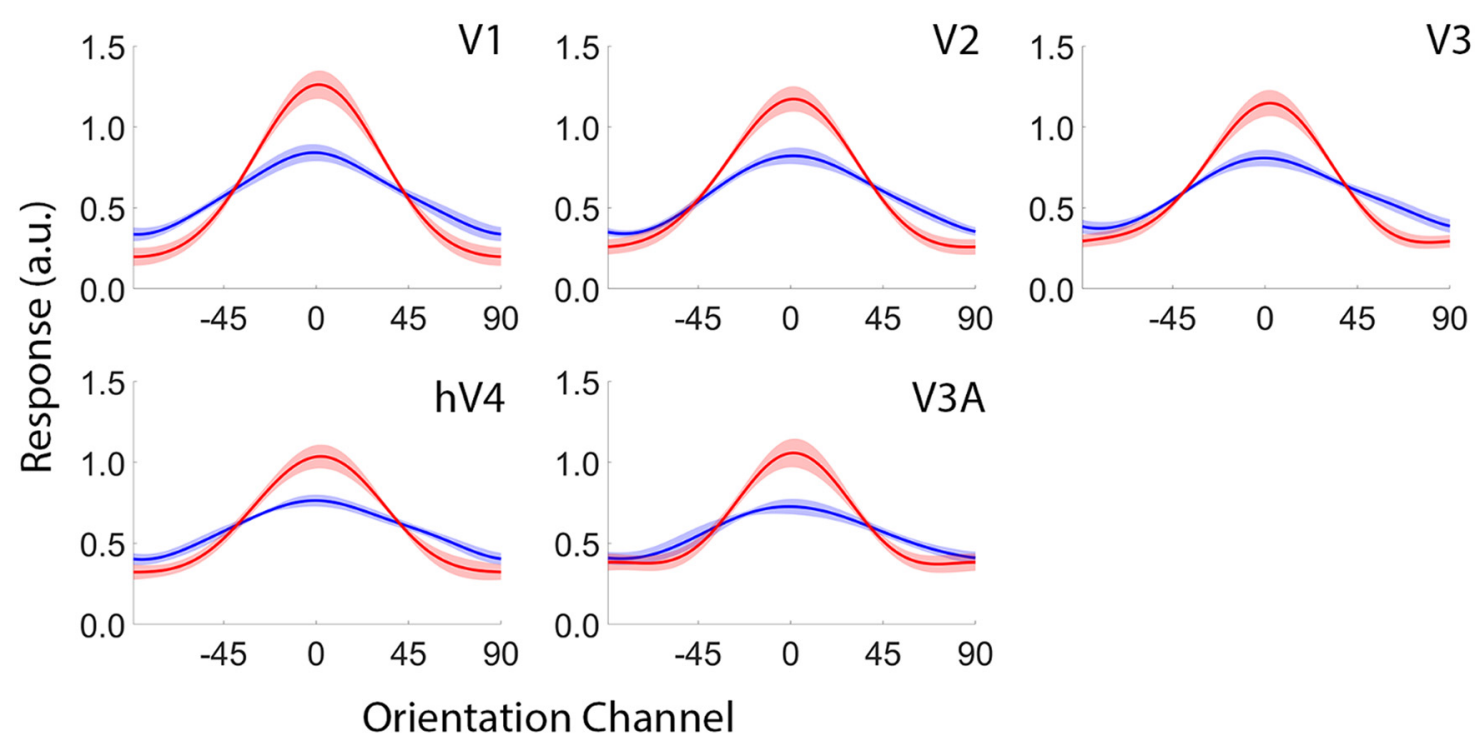

Figure 4. Reconstructions of stimulus orientation during the orientation mapping task (blue) and the category discrimination task (red). Reconstructions were computed using a leave-one-runout cross validation approach where data from $N-1$ runs were used to estimate channel weights and data from the remaining run were used to estimate channel responses. This procedure was iterated until all runs had been used to estimate channel responses, and the results were averaged over permutations. No categorical biases were observed in any visual area for either task. Shaded regions represent \pm 1 within-participant SEM. a.u., Arbitrary units.

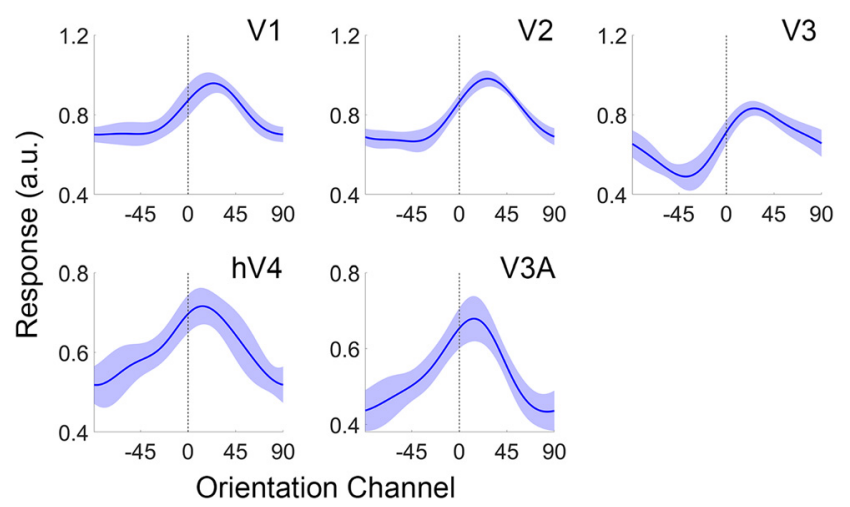

Figure 5. Reconstructed representations of orientation in early visual cortex. The vertical bar at $0^{\circ}$ indicates the actual stimulus orientation presented on each trial. CRFs from Category 1 and Category 2 trials have been arranged and averaged such that any categorical bias would manifest as a clockwise (rightward) shift in the orientation representation toward the center of Category B. Shaded regions represent \pm 1 within-participant SEM (see Materials and Methods). There is a change in scale between visual areas V1-V3 and hV4 -V3A. a.u., Arbitrary units.

that categorical biases in V1-V3 were strongly correlated with variability in overt category judgments (Fig. 8). That is, trial-bytrial category judgments were more strongly associated with the responses of orientation channels near the center of each category rather than those near the physical orientation of the stimulus. Importantly, because the location of the boundary separating Categories 1 and 2 was randomly selected for each participant, it is unlikely that categorical biases shown in Figure 5 reflect intrinsic biases in stimulus selectivity in early visual areas (e.g., due to oblique effects) (Sun et al., 2013).

The category biases shown in Figure 5 may be the result of an adaptive process that facilitates task performance by enhancing the discriminability of physically similar but categorically distinct stimuli. Consider a hypothetical example where an observer is tasked with discriminating between two physically similar exemplars on opposite sides of a category boundary. Discriminating between these alternatives should be challenging as each exemplar evokes a similar and highly overlapping response pattern.
However, discrimination performance could be improved if the responses associated with each exemplar are made more separable via acquired distinctiveness following training (or equivalently, an acquired similarity between exemplars adjacent to the category boundary and exemplars near the center of each category). Similar changes would be less helpful when an observer is tasked with discriminating between physically and categorically distinct exemplars, as each exemplar already evokes a dissimilar and nonoverlapping response. From these examples, a simple prediction can be derived: categorical biases in reconstructed representations of orientation should be largest when participants are shown exemplars adjacent to the category boundary and progressively weaker when participants are shown exemplars further away from the category boundary.

We tested this possibility by sorting stimulus reconstructions according to the angular distance between stimulus orientation and the category boundary (Fig. 9). As predicted, reconstructed representations of orientations adjacent to the category boundary were strongly biased by category membership, with larger biases for exemplars nearest to the category boundary $($ mean $=$ $42.62^{\circ}, 24.16^{\circ}$, and $20.12^{\circ}$ for exemplars located $12^{\circ}, 24^{\circ}$, and $36^{\circ}$ from the category boundary, respectively; FDR-corrected bootstrap, $p<0.0015)$, whereas reconstructed representations of orientations at the center of each category exhibited no signs of bias (mean $=-3.98^{\circ}, p=0.79$; the direct comparison of biases for exemplars adjacent to the category boundary and in the center of each category was also significant; $p<0.01$ ). Moreover, the relationship between average category bias and distance from the category boundary was well approximated by a linear trend (slope $=-14.38^{\circ} / \mathrm{step} ; r^{2}=0.96$ ). Thus, category biases in reconstructed representation are largest under conditions where they would facilitate behavioral performance and absent under conditions where they would not.

Category-selective signals have been identified in multiple brain areas, including portions of lateral occipital cortex, inferotemporal cortex, posterior parietal cortex, and lateral PFC (Freedman et al., 2001; Sigala and Logothetis, 2002; Freedman and Assad, 2006; Pourtois et al., 2009; Folstein et al., 2013; Mack 


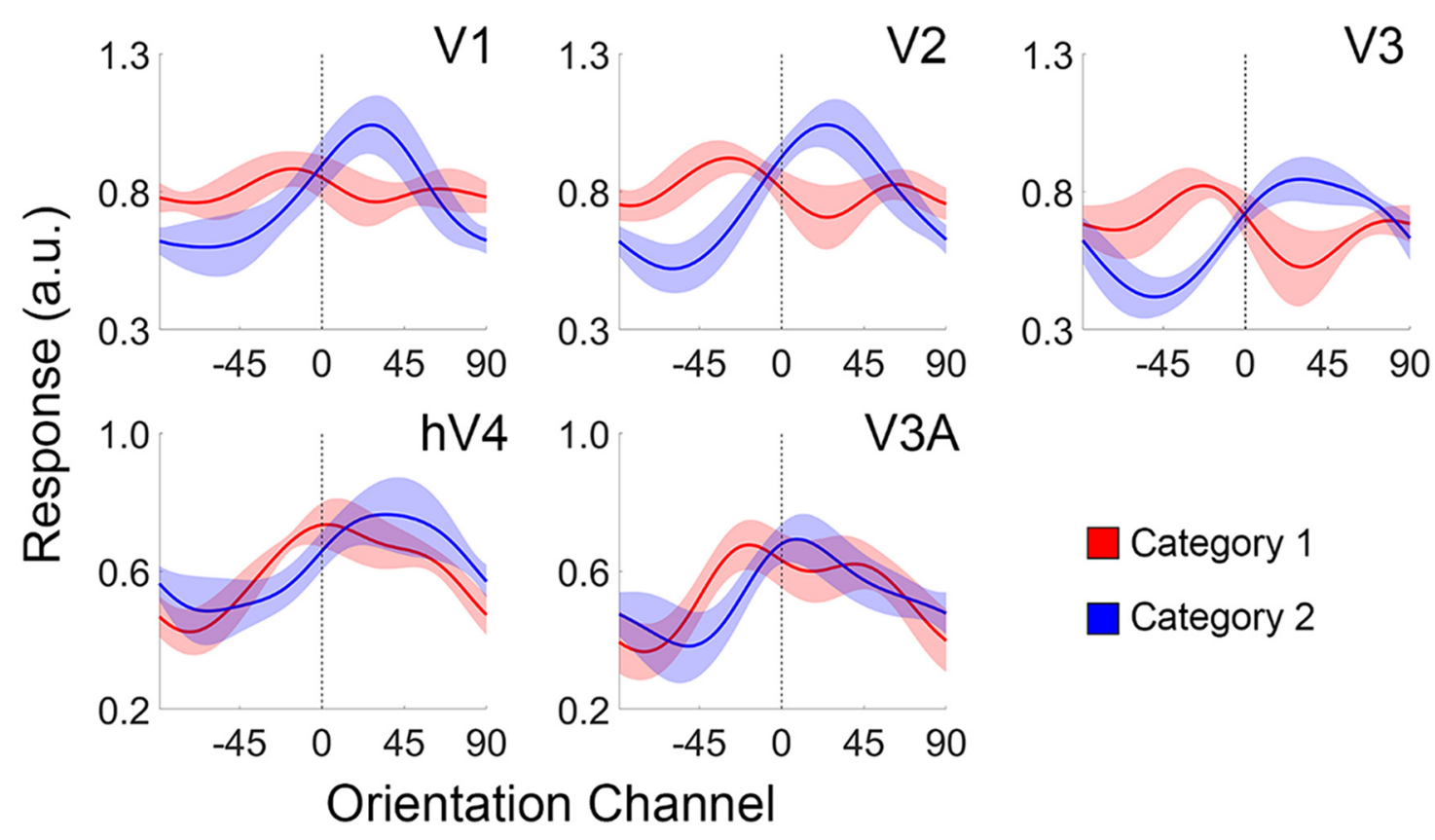

Figure 6. Stimulus reconstructions during Category 1 and Category 2 trials. Shaded regions represent \pm 1 within-participant SEM. a.u., Arbitrary units.

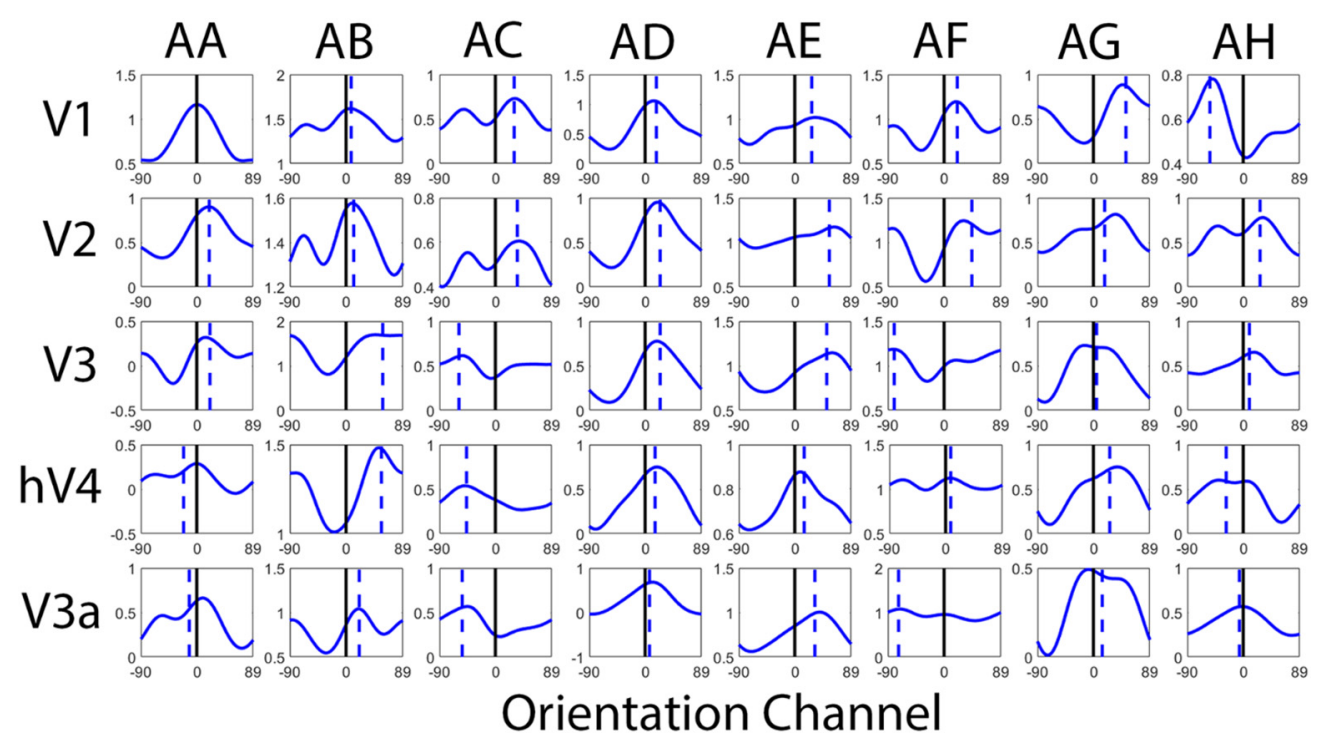

Figure 7. Participant-level stimulus reconstructions. Each panel plots a reconstructed representation of stimulus orientation for a given participant (columns) and visual area (rows). Dashed blue lines indicate the estimated peak of each reconstruction (obtained via curve-fitting). Ordinate units are arbitrary.

et al., 2013; Davis and Poldrack, 2014). We identified categoryselective information in many of these same regions using a whole-brain searchlight-based decoding analysis where a classifier was trained to discriminate between exemplars from Category 1 and Category 2 (independently of stimulus orientation; Fig. 10; see Materials and Methods). Next, we used the same inverted encoding model described above to reconstruct representations of stimulus orientation from activation patterns measured in each area during each of the orientation mapping and category discrimination tasks (reconstructions were computed using a leave-one-participant-out cross-validation routine to ensure that reconstructions were independent of the decoding analysis used to define category-selective ROIs). We were able to reconstruct representations of stimulus orientation in many of these regions during the category discrimination task, but not during the orientation mapping task (where stimulus orientation was task-irrelevant; Fig. 11). This is perhaps unsurprising as representations in many mid- to high-order cortical areas are strongly task-dependent (e.g., Silver et al., 2005). As our analytical approach requires an independent and unbiased estimate of each voxel's orientation selectivity (e.g., during the orientation mapping task), this meant that we were unable to probe categorical biases in reconstructed representations in these regions.

\section{Experiment 2: EEG}

Due to the sluggish nature of the hemodynamic response, the category biases shown in Figures 5 and 9 could reflect processes related to decision making or response selection rather than stimulus processing. In a second experiment, we evaluated the temporal dynamics of category biases using EEG. Specifically, we 


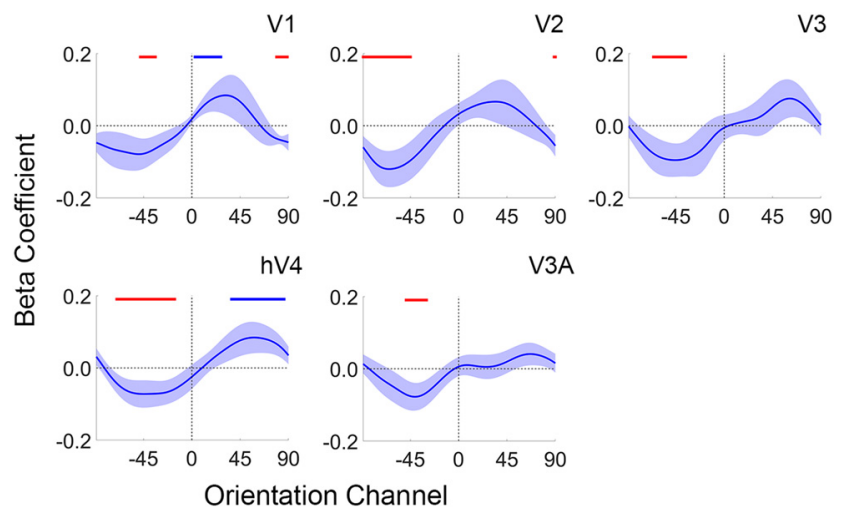

Figure 8. Categorical biases predict choice behavior. Each plot represents a logistic regression of each orientation channel's response onto trial-by-trial variability in category judgments. A positive coefficient indicates a positive relationship between an orientation channel's response and the correct category judgment (i.e., Category B), whereas a negative coefficient indicates a negative relationship between an orientation channel's response and correct category judgment (i.e., Category A). Red and blue horizontal lines at the top of each plot indicate orientation channels whose estimated $\beta$ coefficients are significantly $<0$ or $>0$, respectively (FDR-corrected permutation test; $p<0.05$ ). Shaded regions represent \pm 1 within-participant SEM.

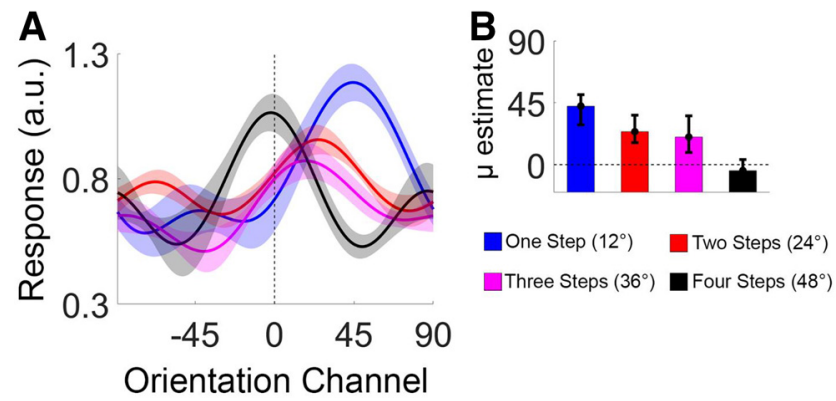

Figure 9. Category biases scale inversely with distance from the category boundary. $\boldsymbol{A}$, The reconstructions shown in Figure 3 sorted by the absolute angular distance between each exemplar and the category boundary. In our case, the 15 orientations were bisected into two groups of 7 , with the remaining orientation serving as the category boundary. Thus, the maximum absolute angular distance between each orientation category and the category boundary was $48^{\circ}$. Participant-level reconstructions were pooled and averaged across visual areas V1, V2, and $\mathrm{V} 3$ as no differences were observed across these regions. Shaded regions represent \pm 1 withinparticipant SEM. $\boldsymbol{B}$, The amount of bias for exemplars located 1, 2, 3, or 4 steps from the category boundary (quantified via a curve-fitting analysis). Error bars indicate $95 \%$ Cls. a.u., Arbitrary units.

reasoned that, if the biases shown in Figures 5 and 9 reflect processes related to decision making, response selection, or motor planning, then these biases should manifest only during a period shortly before the participants' response. Conversely, if the biases are due to changes in how sensory neural populations encode features, they should be evident during the early portion of each trial. To evaluate these alternatives, we recorded EEG while a new group of 28 volunteers performed variants of the orientation mapping and categorization tasks used in the fMRI experiment. On each trial, participants were shown a large annulus of isooriented bars that flickered at $30 \mathrm{~Hz}$ (i.e., $16.67 \mathrm{~ms}$ on, $16.67 \mathrm{~ms}$ off; Fig. 12A). During the orientation mapping task, participants detected and reported the identity of a target letter (an X or a Y) that appeared in a rapid series of letters over the fixation point. Identical displays were used during the category discrimination task, with the caveat that participants were asked to report the category of the oriented stimulus while ignoring the letter stream.

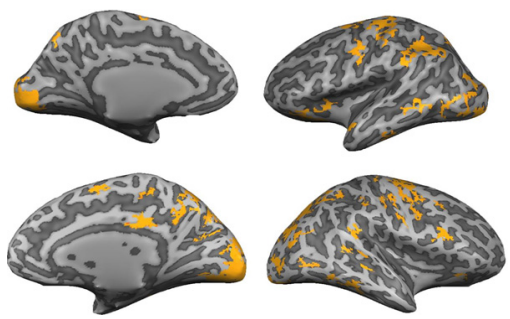

Figure 10. Cortical areas supporting robust decoding of category information. We trained a linear support vector machine to discriminate between activation patterns associated with Category A and Category B exemplars (see Searchlight classification analysis). The trained classifier revealed robust category information in multiple visual, parietal, temporal, and prefrontal cortical areas, including many regions previously associated with categorization (e.g., posterior parietal cortex and lateral PFC).

Mapping Task

Category Task

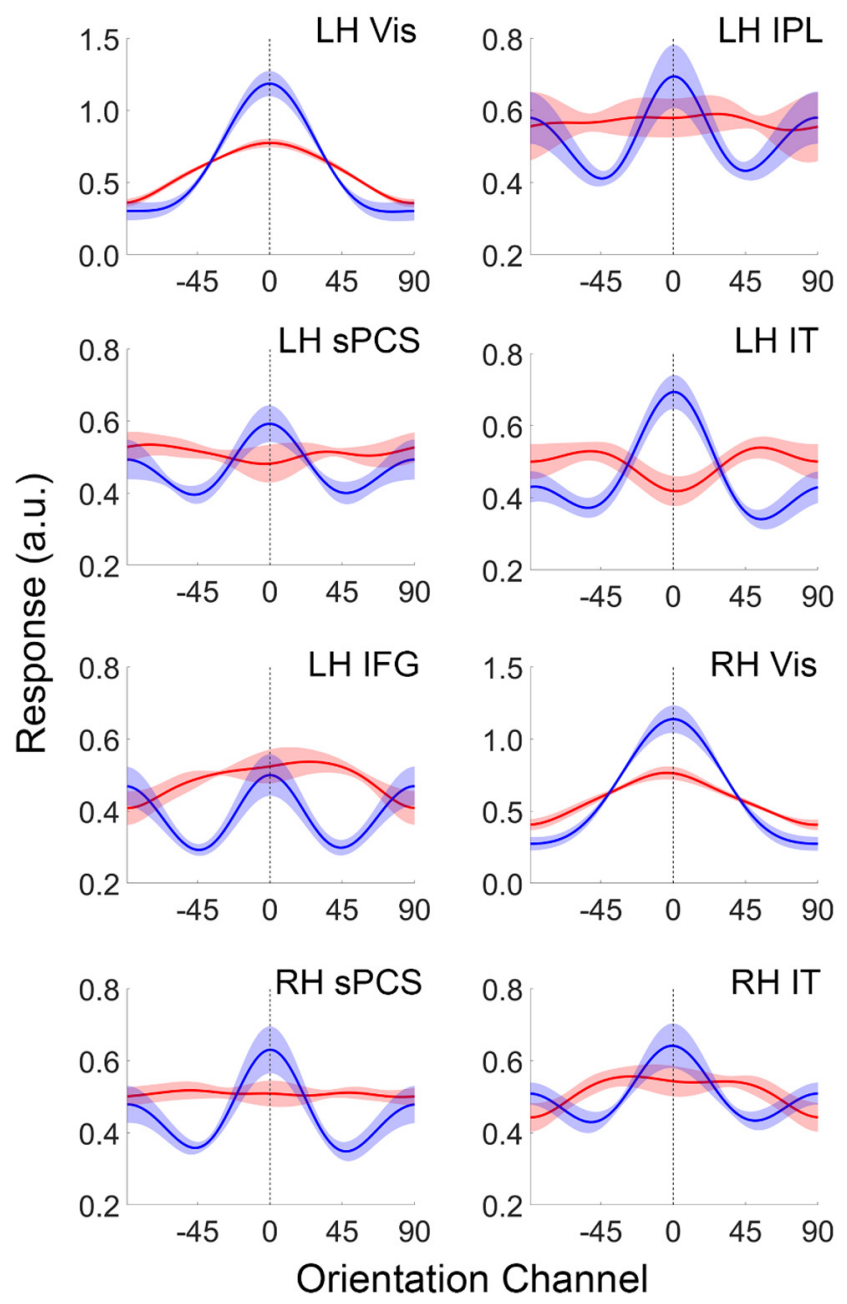

Figure 11. Stimulus reconstructions in visual, parietal, and frontal cortical areas during the orientation mapping and categorization tasks. During the orientation mapping task, participants detected and reported the identity of a target presented in a stream of letters at fixation. During the categorization experiment, participants categorized stimulus orientationintotwo discretegroups. Shaded regions represent \pm 1 withinparticipant SEM. IPL, Inferior parietal lobule; IPS, intraparietal sulcus; ;PCS, superior precentral sulcus; IT, inferotemporal cortex, IFG, inferior frontal gyrus; a.u., arbitrary units.

The $30 \mathrm{~Hz}$ flicker of the oriented stimulus elicits a standing wave of frequency-specific sensory activity known as a steadystate visually evoked potential (SSVEP; Fig. 12B) (Vialatte et al., 2010). The coarse spatial resolution of EEG precludes precise 


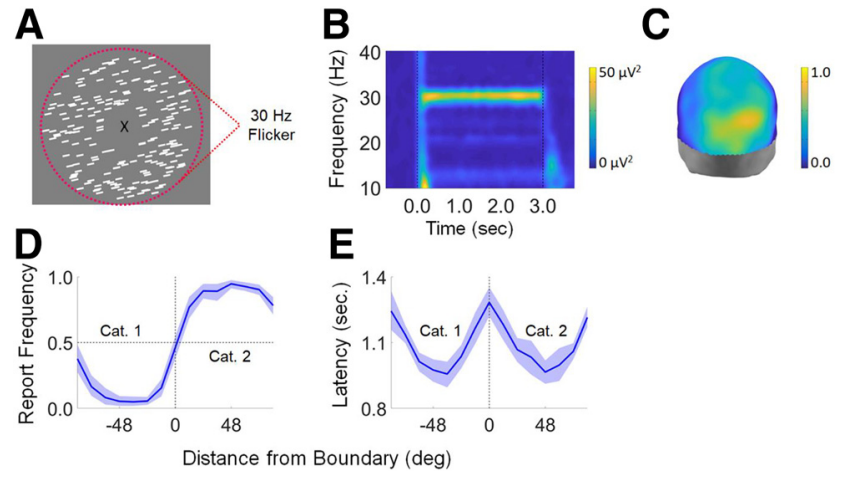

Figure 12. Summary of Experiment 2. A, Participants viewed displays containing an aperture of iso-oriented bars flickering at $30 \mathrm{~Hz}$. $\boldsymbol{B}$, The $30 \mathrm{~Hz}$ flicker entrained a frequency-specific response known as a SSVEP. C, Evoked $30 \mathrm{~Hz}$ power was largest over occipitoparietal electrode sites. We computed stimulus reconstructions (Fig. 7) using the 32 scalp electrodes with the highest power. Scale bar: the proportion of participants (of 27) for which each electrode site was ranked in the top 32 of all 128 scalp electrodes. D, E, Participants categorized stimuli with a high degree of accuracy; incorrect and slow responses were observed only for exemplars adjacent to a category boundary. Shaded regions represent \pm 1 within-participant SEM.
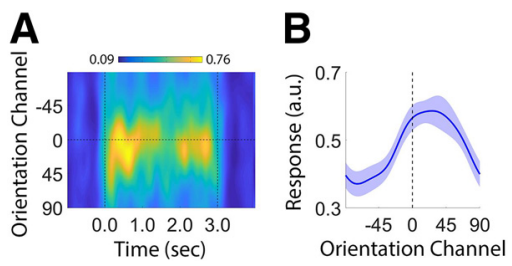

Figure 13. Category biases emerge shortly after stimulus onset. A, Time-resolved reconstruction of stimulus orientation. Dashed vertical lines at time 0.0 and $3.0 \mathrm{~s}$ indicate stimulus onset and offset, respectively. $\boldsymbol{B}$, Average (RF during the first $250 \mathrm{~ms}$ of each trial. The reconstructed representation exhibits a robust category bias ( $p<0.01$; bootstrap test). a.u., Arbitrary units.

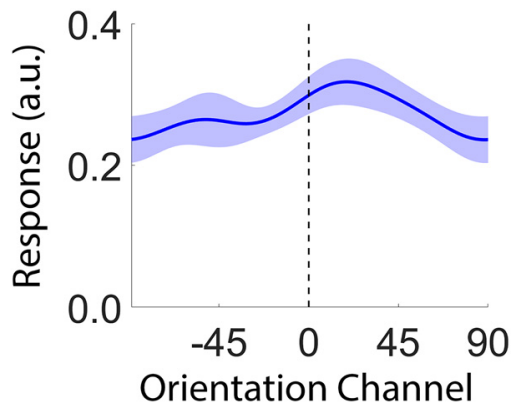

Figure 14. Stimulus and category information is absent in pretrial EEG activity. Timeaveraged reconstruction computed over an interval spanning -250 to 0 ms relative to stimulus onset. The center of the reconstruction was statistically indistinguishable from $0^{\circ}(p=0.234$; bootstrap test).

statements about the cortical source(s) of these signals (e.g., V1, $\mathrm{V} 2$, etc.). However, to focus on visual areas (rather than parietal or frontal areas), we deliberately entrained stimulus-locked activity at a relatively high frequency $(30 \mathrm{~Hz})$. Our approach was based on the logic that coupled oscillators can only be entrained at high frequencies within small local networks, whereas larger or more distributed networks can only be entrained at lower frequencies due to conduction delays (Breakspear et al., 2010). Indeed, a topographic analysis showed that evoked $30 \mathrm{~Hz}$ activity was strongest over a localized region of occipitoparietal electrode sites (Fig. 12C). As in Experiment 1, participants learned to cat- egorize stimuli with a high degree of accuracy, with errors and slow responses present only for exemplars adjacent to a category boundary (Fig. 12D,E).

We computed the power and phase of the $30 \mathrm{~Hz}$ SSVEP response across each $3000 \mathrm{~ms}$ trial and then used these values to reconstruct a time-resolved representation of stimulus orientation (Garcia et al., 2013). Our analysis procedure followed that used in Experiment 1: In the first phase of the analysis, we rankordered scalp electrodes by $30 \mathrm{~Hz}$ power (based on a discrete Fourier transform spanning the $3000 \mathrm{~ms}$ trial epoch, averaged across all trials of both the orientation mapping and category discrimination tasks). Responses measured during the orientation mapping task were used to estimate a set of orientation weights for the 32 electrodes with the strongest SSVEP signals (i.e., those with the highest $30 \mathrm{~Hz}$ power; see Fig. 12C) at each time point. In the second phase of the analysis, we used these time point-specific weights and corresponding responses measured during each trial of the category discrimination task across all electrodes to compute a time-resolved representation of stimulus orientation (Fig. 13A,B). We reasoned that, if the categorical biases shown in Figures 5 and 9 reflect processes related to decision making or response selection, then they should emerge gradually over the course of each trial. Conversely, if the categorical biases reflect changes in sensory processing, then they should manifest shortly after stimulus onset. To test this possibility, we computed a temporally averaged stimulus reconstruction over an interval spanning 0-250 ms after stimulus onset (Fig. 14B). A robust category bias was observed (mean $23.35^{\circ} ; p=0.014$; bootstrap test), suggesting that the intent to categorize a stimulus modulates how neural populations in early visual areas respond to incoming sensory signals.

Importantly, the bandpass filter used to isolate $30 \mathrm{~Hz}$ activity will distort temporal characteristics of the raw EEG signal by some amount. We estimated the extent of this distortion by generating a $3 \mathrm{~s}, 30 \mathrm{~Hz}$ sinusoid with unit amplitude (plus $1 \mathrm{~s}$ of presignal and postsignal zero padding) and running it through the same filters used in our analysis path. We then computed the time at which the filtered signal reach $25 \%$ of maximum. For an instantaneous filter, this should occur at exactly $1 \mathrm{~s}$ (due to the presignal and postsignal zero padding). We estimated a signal onset of $\sim 877 \mathrm{~ms}$, or $123 \mathrm{~ms}$ before the start of the signal. Therefore, if reconstruction amplitude is $>0$ at time $t$, then we can conclude that the pattern of scalp activity used to generate the stimulus reconstruction contained reliable orientation information at time $t \pm 125 \mathrm{~ms}$. The same logic applies to estimates of reconstruction bias as the reconstructions are based on data filtered using the same parameters. Importantly, we also verified that there was no categorical bias in stimulus reconstructions before stimulus onset (Fig. 14), nor were categorical biases present when we reconstructed stimulus representations using data from the orientation mapping and category discrimination tasks separately (Fig. 15).

\section{Ruling out contributions from eye movements}

We identified and removed trials contaminated by large EOG artifacts (blinks and eye movements $\gtrsim 2^{\circ}$ ). However, small and consistent eye movement patterns could nevertheless contribute to the orientation reconstructions reported here. We examined this possibility by testing whether participants foveated the inner aperture of the stimulus at polar locations matching its orientation (Fig. 16A) or at polar locations matching the center of the appropriate category (A vs B; Fig. 16B; for details, see Materials and Methods). No systematic differences in eye position were 


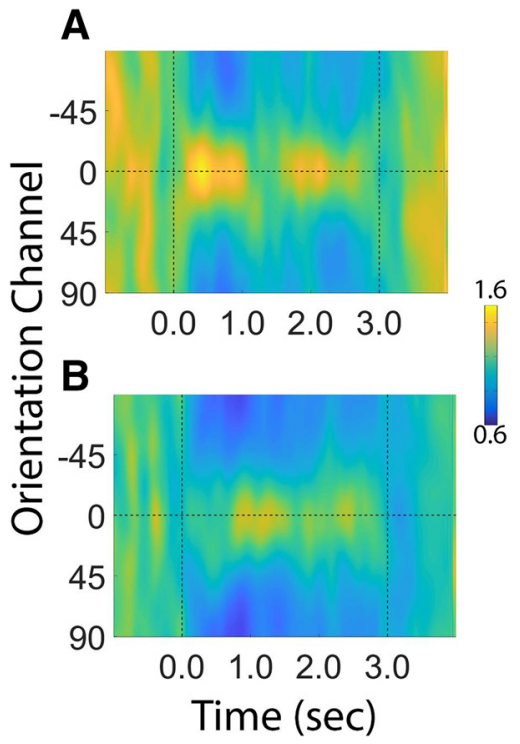

Figure 15. Reconstructions of stimulus orientation during the orientation mapping task ( $\boldsymbol{A})$ and the category discrimination task $(\boldsymbol{B})$ during Experiment 2. Vertical dashed lines at time 0.0 and 3.0 indicate the start and end of each trial, respectively. Reconstructions were computed using a leave-one-run-out cross validation approach where data from $N-1$ runs were used to estimate channel weights and data from the remaining run were used to estimate channel responses. This procedure was iterated until all runs had been used to estimate channel responses, and the results were averaged over permutations. Units of response are arbitrary.

observed as a function of stimulus orientation or category membership (Fig. 16), suggesting that eye movements were not a major contributor to orientation-specific reconstructions.

\section{Experiment 3: EEG}

The results of Experiments 1 and 2 suggest that category learning can bias stimulus-specific representations encoded by occipitoparietal cortical areas. However, an alternative explanation is that the biases shown in Figures 5, 9, and 13 reflect mechanisms of response selection or decision making independent of categorical processing. Experiment 3 examined this possibility by examining categorical biases in stimulus-specific memory representations while participants performed a DMC task. A schematic of the task is shown in Figure 17A, B. At the beginning of each trial a sample disc rendered in one of 12 possible stimulus locations (15-345 polar angle in $30^{\circ}$ along the perimeter of an imaginary circle). Half of the disc positions were assigned membership in Category 1 , while the remaining half of disc positions were assigned membership in Category 2 (Fig. 17A). Participants remembered the position of the sample disc over a blank delay, then judged whether a probe disc was rendered in a position matching the category of the sample disc. The location of the category boundary was randomly determined for each participant, and response feedback (correct vs incorrect) was provided after every trial. Like Experiment 2, participants were not trained on the DMC task before testing and learned to associate specific positions with specific categories through feedback. Before completing the DMC task, participants also completed a spatial working memory task. Display and stimulus geometry were identical during the spatial memory task and the DMC task. On each trial a sample disc was rendered in one of the same 12 positions used during the DMC task. After a short delay, participants recalled the location of the sample disc via mouse click.

Following earlier work (Samaha et al., 2016; e.g., Foster et al., 2016; Ester et al., 2018; Nouri and Ester, 2020), we used spatio-

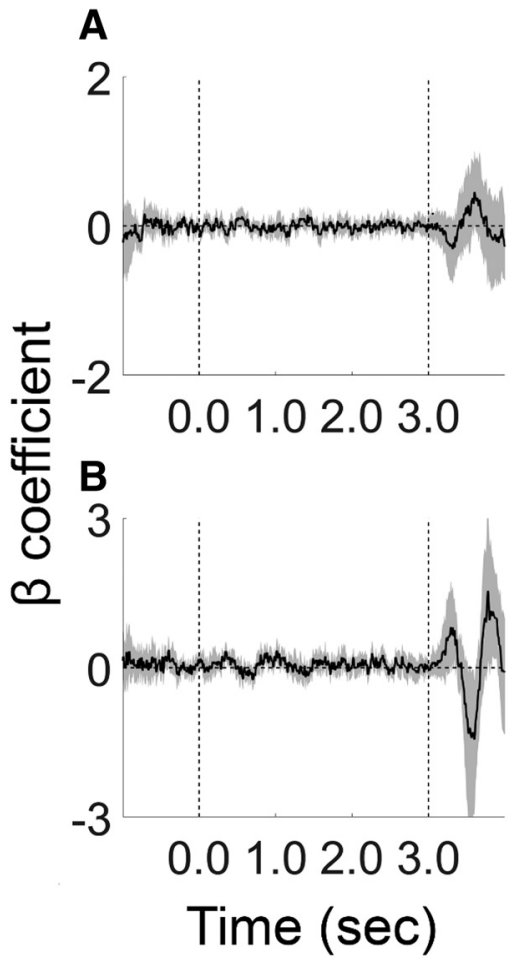

Figure 16. No systematic biases in eye position during orientation categorization (Experiment 2). We regressed trial-by-trial records of stimulus orientation $(\boldsymbol{A})$ or category $(\boldsymbol{B})$ onto horizontal EOG activity. Thus, positive coefficients reflect a systematic relationship between stimulus orientation (or category) and eye position. No such biases were observed. Black vertical dashed lines at 0.0 and 3.0 indicate the start and end of each trial, respectively. Shaded regions represent the $95 \%$ within-participant $\mathrm{Cl}$ of the mean.

temporal patterns of induced alpha-band $(8-12 \mathrm{~Hz})$ activity over occipitoparietal electrode sites to track the contents of spatial working memory during the recall and DMC tasks. Specifically, we modeled sample-by-sample estimates of alpha band activity recorded during the spatial recall task as a combination of 12 location filters, each with an idealized tuning curve (a cosine raised to the $12^{\text {th }}$ power). The result of this step is a set of weights that characterizes the location preferences of each scalp electrode. Next, we used these weights and spatiotemporal patterns of alpha-band activity recorded during the DMC task to compute an expected response for each location filter, yielding a timeresolved estimate of stimulus position. Trial-by-trial response functions were shifted to a common center $\left(0^{\circ}\right.$ by convention), averaged, and arranged such that any category bias would manifest as a clockwise or positive shift toward the center of Category 2.

As expected, a robust category bias was observed during the delay period of the DMC task (Fig. 17C), though unlike Experiment 2 the bias seemed to emerge gradually over the course of the delay period. To quantify this bias, we averaged channel responses from period 0.25 to $2.0 \mathrm{~s}$ after onset of the sample display and fit the resulting function with an exponentiated cosine (see Quantification of bias in orientation representations). Mean reconstruction centers were reliably $>0^{\circ}$ (mean $10.55^{\circ} ; p=0.002$, bootstrap test), indicating a robust bias toward the center of the relevant category. Importantly, this bias cannot be explained by mechanisms associated with decision making and response selection: participants could not plan or implement a response until the probe stimulus was presented at the end of the delay period. This result further suggests that the results of Experiments 1 and 2 cannot be wholly explained by mechanisms of response selection or bias. 


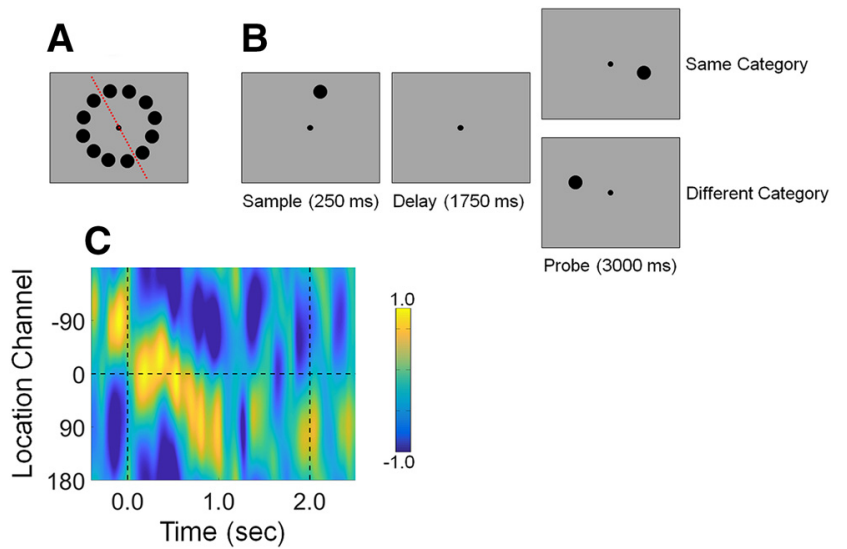

Figure 17. Design and results of Experiment 3.A, Possible stimulus locations. The orientation of the category boundary (red dashed line) was randomly determined for each participant (example shown). $\boldsymbol{B}$, DMC task. Participants remembered the position of a sample disc over a blank delay and then judged whether the location of a probe disc was drawn from the same location category or a different location category. In this example, the categories are defined by the boundary shown in $A$. C, Location-specific reconstructions computed during the DMC task. Vertical dashed lines at 0.0 and $2.0 \mathrm{~s}$ indicate the onset of the sample and probe epochs, respectively. Participants could not prepare a response until the onset of the probe display, yet a robust category bias was observed during the delay period. This suggests that category biases observed in Experiments 1 and 2 are not solely due to mechanisms of response selection.

Assessing contributions from eye movements

We identified and removed EOG artifacts from the data via independent components analysis. However, small and consistent eye movement patterns opaque to independent components analysis could nevertheless contribute to the location reconstructions reported here. We examined this possibility by regressing timeresolved estimates of horizontal EOG activity onto remembered stimulus locations. As shown in Figure 18, the regression coefficients linking eye position with remembered locations were indistinguishable from 0 for the duration of each trial, suggesting that eye movements were not a major determinant of location reconstructions.

\section{Discussion}

Our findings suggest that category learning shapes information processing at the earliest stages of the visual system. The results of Experiment 1 showed that representations of a to-be-categorized stimulus encoded by population-level activity in early visual cortical areas were systematically biased by their category membership. These biases were correlated with overt category judgments and were largest for exemplars adjacent to the category boundary. The results of Experiments 2 and 3 demonstrate that similar biases are present in orientation- and location-specific reconstructions computed by human scalp EEG data, and further suggest that our findings cannot be explained by response bias, motor planning, or eye movements.

The categorical biases reported here are strongly task-dependent, and therefore must reflect changes in responses caused by transient top-down factors rather than long-term changes in feature or location selectivity. However, the effects of these top-down modulations are fundamentally different from task-dependent modulations reported elsewhere. In one example, Ester et al. (2015) asked participants to attend the orientation or luminance of a peripheral grating and found both multiplicative and additive enhancements of orientation-specific reconstructions during the attend orientation condition relative to the attend luminance condition, but no evidence for a shift like the one reported here.

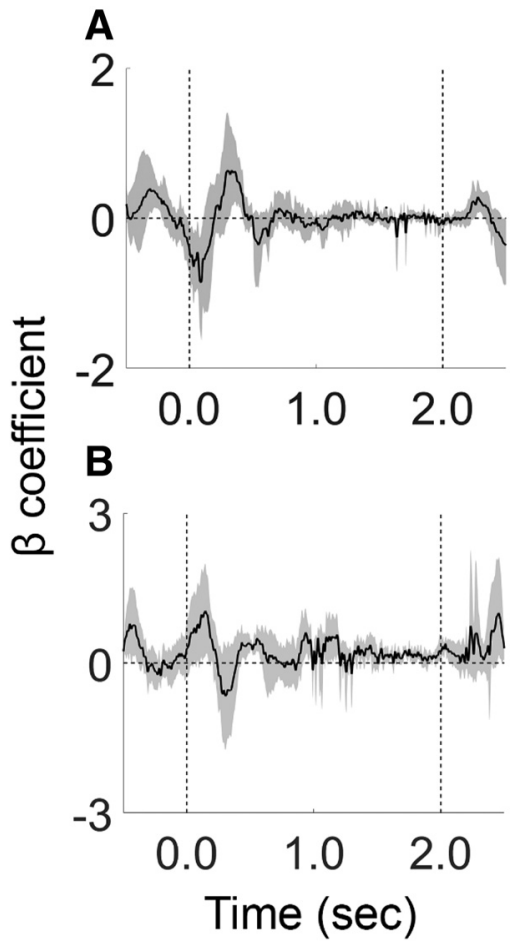

Figure 18. No systematic biases in eye position during location categorization (Experiment 3). We regressed trial-by-trial records of stimulus location $(\boldsymbol{A})$ or category $(\boldsymbol{B})$ onto horizontal EOG activity. Thus, positive coefficients reflect a systematic relationship between stimulus orientation (or category) and eye position. No such biases were observed. Black vertical dashed lines at 0.0 and 3.0 indicate the start and end of each trial. Shaded regions represent the $95 \%$ within-participant $\mathrm{Cl}$ of the mean.

In a different study, Byers and Serences (2014) examined changes in orientation-specific reconstructions before and after participants underwent extensive training (10 $1 \mathrm{~h}$ sessions) in a challenging orientation discrimination task. We observed changes in the amplitude (i.e., signal-to-noise ratio) of orientation-specific reconstructions following training, but no evidence for a shift like the one reported in the current study. In other studies, Scolari et al. (2012) examined changes in orientation-specific reconstructions when participants performed fine-grained and coarsegrained orientation discrimination tasks. Participants viewed two oriented gratings in sequence and judged whether they were identical. During one experiment participants were cued to how the second grating might differ from the first (clockwise vs counterclockwise rotation), whereas in a second experiment they were not. During the fine-grained discrimination task, the authors observed a modest shift in orientation-specific reconstructions toward "off-target" neural populations that maximally discriminated between two oriented stimuli, but only when participants were cued to expect a clockwise or counterclockwise rotation. While this type of modulation is desirable while performing a fine-discrimination task, it is qualitatively different from the shifts we report in the current experiment, as participants have no way of anticipating what orientation will be presented on each trial, nor the difference between that orientation and the category boundary. Moreover, the shifts reported by Scolari et al. (2012) during fine discriminations were relatively modest, at most few degrees. We report an opposite pattern of findings, where shifts are largest for oriented exemplars immediately adjacent to the category boundary. Thus, while other studies have documented task-dependent changes in orientation-specific reconstructions, 
those studies have failed to reveal shifts in reconstructed representations (Byers and Serences, 2014; Ester et al., 2015) or have revealed modest shifts that follow different patterns from those reported here (Scolari et al., 2012).

Several mechanisms may be responsible for our findings. One possibility is that the orientation preferences of single units (or populations of units) are systematically shifted toward the center of each category during the category discrimination task, much in the same way that neurons in the rodent auditory system exhibit emergent selectivity for categorically different stimuli (e.g., Xin et al., 2019) or in the same way that the spectral preferences of neural populations are biased by feature-based attention (David et al., 2008; Cukur et al., 2013). These shifts are relatively small at the single-unit level but could be amplified by read-out mechanisms that integrate the responses of large neural populations. A second possibility is that participants strategically apply gain to neural populations that maximally discriminate between to-becategorized exemplars during the category discrimination task. Here there are no changes in the spectral preferences of single units, but the responses of neurons that respond to stimuli near the category boundary are amplified. These alternatives are not mutually exclusive; nor is this an exhaustive list. Our data cannot resolve these possibilities. For example, several different patterns of single-unit gain changes and/or tuning shifts can produce identical responses in a single fMRI voxel, and different patterns of single-voxel modulation could produce categorical biases in multivariate stimulus reconstructions (for a detailed discussion of this issue, see, e.g., Sprague et al., 2018). Ultimately, targeted experiments that combine noninvasive measurements of brain activity with careful psychophysical measurements and detailed model simulations will be needed to conclusively identify the mechanisms responsible for the category biases we have reported here.

Our findings appear to conflict with results from nonhuman primate research, which suggests that sensory cortical areas do not encode categorical information. However, there is reason to suspect that mechanisms of category learning might be qualitatively different in human and nonhuman primates. For example, our participants learned to categorize stimuli based on performance feedback after $\sim 10 \mathrm{~min}$ of training. In contrast, macaque monkeys typically require 6 months or more of training using a similar feedback scheme to reach a similar level of performance, and this extensive amount of training may influence how neural circuits code information (Birman and Gardner, 2016; e.g., Itthipuripat et al., 2017). Moreover, there is growing recognition that the contribution(s) of sensory cortical areas to performance on a visual task are highly susceptible to recent history and training effects (Chen et al., 2016; Itthipuripat et al., 2017; Liu and Pack, 2017). In one example (Liu and Pack, 2017), extensive training was associated with a functional substitution of human visual area $\mathrm{V} 3 \mathrm{a}$ for $\mathrm{MT}^{+}$in discriminating noisy motion patches. Thus, training effects may help explain why previous electrophysiological experiments have found category-selective responses in association but not sensory cortical areas.

Studies of categorization in nonhuman primates have typically used variants of a delayed match to category task, where monkeys are shown a sequence of two exemplars separated by a blank delay interval and asked to report whether the category of the second exemplar matches the category of the first exemplar. The advantage of this task is that it allows experimenters to decouple category-selective signals from activity related to decision making, response preparation, and response execution. However, this same advantage also precludes examinations of whether and/or how top-down category-selective signals interact with bottom-up stimulus-specific signals. We made no effort to decouple category-selective and decision-related signals in Experiments 1 and 2; thus, the category biases observed in those studies could reflect mechanisms of decision making, response selection, or motor planning. The results of Experiment 3 conflict with this interpretation by demonstrating that robust category biases are present during the memory period of a DMC task (Freedman and Assad, 2006).

Previous studies have identified cortical modules selective for faces (Kanwisher et al., 1997), locations (Epstein and Kanwisher, 1998), actions (Astafiev et al., 2004; Huth et al., 2012), bodies (Downing et al., 2001), animacy (Konkle and Caramazza, 2013), and size (Konkle and Caramazza, 2013). Other category distinctions (e.g., tools vs cars) lack specialized processing modules but can be decoded from multivoxel patterns in multiple occipitotemporal regions (e.g., Folstein et al., 2013). Our findings complement these studies by demonstrating that learning a novel and arbitrary category rule is correlated with rapid and reversible changes in stimulus-specific information processing at even earlier stages of the cortical visual processing hierarchy, including V1 (see also Brouwer and Heeger, 2009, 2013). Category-dependent changes in early visual areas may contribute to more complex forms of category selectivity exhibited by upstream cortical areas, including portions of lateral occipital and inferotemporal cortex. This possibility awaits further scrutiny.

In conclusion, we have shown that learning a novel and arbitrary category rule based on a simple visual feature (orientation or location) correlates with rapid and reversible changes in sensory and mnemonic representations encoded by regions in early occipitoparietal cortex. These changes correlate with participants' overt category judgments, are largest for exemplars adjacent to a category boundary, and cannot be explained by decision making or motor preparation. Collectively, these results provide novel and important evidence suggesting that category learning induces rapid-yet-reversable changes in information processing at early stages of the cortical visual processing hierarchy.

\section{References}

Ashby FG, Maddox WT (2005) Human category learning. Annu Rev Psychol 56:148-178.

Astafiev SV, Stanley CM, Shulman GL, Corbetta M (2004) Extrastriate body area in human occipital cortex responds to the performance of motor actions. Nat Neurosci 7:542-548.

Birman D, Gardner JL (2016) Parietal and prefrontal: categorical differences? Nat Neurosci 19:5-7.

Blankertz B, Lemm S, Treder M, Haufe S, Muller KR (2011) Single-trial analysis and classification of ERP components - a tutorial. Neuroimage 56:814-825.

Breakspear M, Heitmann S, Daffertshofer A (2010) Generative models of cortical oscillations: neurobiological implication of the Kuramoto model. Front Hum Neurosci 4:190.

Brouwer GJ, Heeger DJ (2009) Decoding and reconstructing color from responses in human visual cortex. J Neurosci 29:13992-14003.

Brouwer GJ, Heeger DJ (2011) Cross-orientation suppression in human visual cortex. J Neurophysiol 106:2108-2119.

Brouwer GJ, Heeger DJ (2013) Categorical clustering of the neural representation of color. J Neurosci 33:15454-15465.

Byers A, Serences JT (2014) Enhanced attentional gain as a mechanism for generalized perceptual learning in human visual cortex. J Neurophysiol 112:1217-1227.

Chang CC, Lin CJ (2011) LIBSVM: a library for support vector machines. ACM Trans Intell Syst Technol 2:1-27.

Chen N, Cai P, Zhou T, Thompson B, Fang F (2016) Perceptual learning modifies the functional specializations of visual cortical areas. Proc Natl Acad Sci U S A 113:5724-5729.

Cousineau D (2005) Confidence intervals in within-subject designs: a sim- 
pler solution to Loftus and Masson's method. Quant Methods Psychol $1: 42-45$.

Cukur T, Nishimoto S, Huth AG, Gallant JL (2013) Attention during natural vision warps semantic representation across the human brain. Nat Neurosci 16:763-770.

David SV, Hayden BY, Mazer JA, Gallant JL (2008) Attention to stimulus features shifts spectral tuning of V4 neurons during natural vision. Neuron 59:509-521.

Davis T, Poldrack RA (2014) Quantifying the internal structure of categories using a neural typicality measure. Cereb Cortex 24:1720-1737.

Delorme A, Makeig S (2004) EEGLAB: An open source toolbox for analysis of single-trial EEG dynamics including independent component analysis. Journal of Neuroscience Methods 134:9-21.

Downing PE, Jiang Y, Shuman M, Kanwisher N (2001) A cortical area selective for visual processing of the human body. Science 293:2470-2473.

Epstein R, Kanwisher N (1998) A cortical representation of the local visual environment. Nature 392:598-601.

Ester EF, Sprague TC, Serences JT (2015) Parietal and frontal cortex encode stimulus-specific mnemonic representations during visual working memory. Neuron 87:893-905.

Ester EF, Nouri A, Rodriguez L (2018) Retrospective cues mitigate information loss in human cortex during working memory storage. J Neurosci 38:8538-8548.

Esterman M, Tamber-Rosenau BJ, Chiu YC, Yantis S (2010) Avoiding nonindependence in fMRI data analysis: leave one subject out. Neuroimage 50:572-576.

Folstein JR, Palmeri TJ, Gauthier I (2013) Category learning increases discriminability of relevant object dimensions in visual cortex. Cereb Cortex 23:714-823.

Foster JJ, Sutterer DW, Serences JT, Vogel EK, Awh E (2016) The topography of alpha-band activity tracks the content of spatial working memory. J Neurophysiol 115:168-177.

Freedman DJ, Assad JA (2006) Experience-dependent representation of visual categories in parietal cortex. Nature 443:85-88.

Freedman DJ, Riesenhuber M, Poggio T, Miller EK (2001) Categorical representation of visual stimuli in the primate prefrontal cortex. Science 291:312-316.

Garcia JO, Srinivasan R, Serences JT (2013) Near-real-time feature-selective modulations in human cortex. Curr Biol 23:515-522.

Goldstone R (1994) Influence of categorization on perceptual discrimination. J Exp Psychol Gen 123:178-200.

Goldstone RL (1998) Perceptual learning. Annu Rev Psychol 49:585-612.

Huth AG, Nishimoto S, Vu AT, Gallant JL (2012) A continuous semantic space describes the representation of thousands of object and action categories across the human brain. Neuron 76:1210-1224.

Itthipuripat S, Cha K, Byers A, Serences JT (2017) Two different mechanisms support selective attention at different phases of training. PLoS Biol 15:e2001724.

Kanwisher N, McDermott J, Chun MM (1997) The fusiform face area: a module in human extrastriate cortex specialized for face perception. J Neurosci 17:4302-4311.
Kleiner M, Brainard D, Pelli D (2007) What's new in Psychtoolbox-3. Perception 36:14.

Kok P, Mostert P, De Lange, FP (2017) Prior expectations induce prestimulus sensory templates. Proceedings of the National Academy of Sciences USA 114:10473-10478.

Konkle T, Caramazza A (2013) Tripartite organization of the ventral stream by animacy and object size. J Neurosci 33:10235-10242.

Lins OG, Picton TW, Berg P, Scherg M (1993) Ocular artifacts in EEG and event-related potentials 1: Scalp topograpy. Brain Topography 6:51-63.

Liu LD, Pack CC (2017) The contribution of area MT to visual motion perception depends on training. Neuron 95:436-446.e3.

Livingston KR, Andrews JK, Harnad S (1998) Categorical perception effects induced by category learning. J Exp Psychol Learn Mem Cogn 24:732753.

Mack ML, Preston AR, Love BC (2013) Decoding the brain's algorithm for categorization from its neural implementation. Curr Biol 23:2023-2027.

Mostert P, Albers AM, Brinkman L, Todorova L, Kok P, de Lange FP (2018) Eye movement-related confounds in neural decoding of visual working memory representations. eNeuro 5: ENEURO.0401-17.2018.

Newell FN, Bülthoff HH (2002) Categorical perception of familiar objects. Cognition 85:113-143.

Nouri A, Ester EF (2020) Recovery of information from latent memory stores decreases over time. Cogn Neurosci 11:101-110.

Pourtois G, Schwartz S, Spiridon M, Martuzzi R, Vuilleumier P (2009) Object representations for multiple visual categories overlap in lateral occipital and medial fusiform cortex. Cereb Cortex 19:1806-1819.

Quax SC, Dijkstra N, van Staveren MJ, Bosch SE, van Gerven MAJ (2019) Eye movements explain decodability during perception and cued attention in MEG. Neuroimage 195:444-453.

Samaha J, Sprague TC, Postle BR (2016) Decoding and reconstructing the focus of spatial attention from the topography of alpha-band oscillations. Journal of Cognitive Neuroscience 29:1090-1097.

Scolari M, Byers A, Serences JT (2012) Optimal deployment of attentional gain during fine discriminations. J Neurosci 32:7723-7733.

Sigala N, Logothetis NK (2002) Visual categorization shapes feature selective in the primate temporal cortex. Nature 415:318-320.

Silver MA, Ress D, Heeger DJ (2005) Topographic maps of visual spatial attention in human parietal cortex. J Neurophysiol 94:1358-1371.

Sprague TC, Adam KC, Foster JJ, Rahmati M, Sutterer DW, Vo VA (2018) Inverted encoding models assay population-level stimulus representations, not single-unit neural tuning. eNeuro 5:ENEURO.0098-18. 2018.

Sun P, Gardiner JL, Costagli M, Ueno K, Waggoner RA, Tanaka K, Cheng K (2013) Demonstration of tuning to stimulus orientation in the human cortex: a high-resolution fMRI study with a novel continuous stimulation paradigm. Cereb Cortex 23:1618-1629.

Vialatte FB, Maurice M, Dauwels J, Cichocki A (2010) Steady-state visually evoked potentials: focus on essential paradigms and future perspectives. Prog Neurobiol 90:418-438.

Xin Y, Zhong L, Zhang Y, Zhou T, Pan J, Xu N-1 (2019) Sensory-to-category transformation via dynamic reorganization of ensemble structures in mouse auditory cortex. Neuron 103:909-921. 